PASJ: Publ. Astron. Soc. Japan , 1-??, 〈publication date〉

(C) 2018. Astronomical Society of Japan.

\title{
X-Ray Study of the Outer Region of Abell 2142 with Suzaku *
}

\author{
H. Akamatsu ${ }^{1}$, A. Hoshino ${ }^{2}$, Y. Ishisaki ${ }^{1}$, T. Ohashi ${ }^{1}$, K. Sato ${ }^{3}$, Y. Takei ${ }^{4}$, N. Ota ${ }^{5}$ \\ ${ }^{1}$ Department of Physics, Tokyo Metropolitan University, \\ 1-1 Minami-Osawa, Hachioji, Tokyo 192-0397 \\ ${ }^{2}$ Graduate School of Natural Science and Technology, \\ Kanazawa University, Kakuma, Kanazawa, Ishikawa 920-1192 \\ ${ }^{3}$ Department of Physics, Tokyo University of Science, \\ 1-3 Kagurazaka, Shinjyuku-ku, Tokyo, 162-8601, Japan \\ ${ }^{4}$ Department of High Energy Astrophysics, Institute of Space and Astronautical Science, \\ Japan Aerospace Exploration Agency, \\ 3-1-1 Yoshinodai, Chuo-ku, Sagamihara, Kanagawa 252-5210 \\ ${ }^{5}$ Nara Women's University, Kitauoyanishi-machi, Nara, Nara 630-8506 \\ h_aka@phys.se.tmu.ac.jp \\ (Received $\langle$ reception date $\rangle$; accepted $\langle$ acception date $\rangle)$
}

\begin{abstract}
We observed outer regions of a bright cluster of galaxies A2142 with Suzaku. Temperature and brightness structures were measured out to the virial radius $\left(r_{200}\right)$ with good sensitivity. We confirmed the temperature drop from $9 \mathrm{keV}$ around the cluster center to about $3.5 \mathrm{keV}$ at $r_{200}$, with the density profile well approximated by the $\beta$ model with $\beta=0.85$. Within $0.4 r_{200}$, the entropy profile agrees with $r^{1.1}$, as predicted by the accretion shock model. The entropy slope becomes flatter in the outer region and negative around $r_{200}$. These features suggest that the intracluster medium in the outer region is out of thermal equilibrium. Since the relaxation timescale of electron-ion Coulomb collision is expected to be longer than the elapsed time after shock heating at $r_{200}$, one plausible reason of the low entropy is the low electron temperature compared to that of ions. Other possible explanations would be gas clumpiness, turbulence and bulk motions of ICM. We also searched for a warm-hot intergalactic medium around $r_{200}$ and set an upper limit on the oxygen line intensity. Assuming a line-of-sight depth of $2 \mathrm{Mpc}$ and oxygen abundance of 0.1 solar, the upper limit of an overdensity is calculated to be 280 or 380 , depending on the foreground assumption.
\end{abstract}

Key words: galaxies: clusters: individual (Abell 2142) - X-rays: galaxies: clusters - X-rays: ICM, WHIM (Warm Hot Intergaractic medium)

\section{Introduction}

The Cold Dark Matter scenario of the cosmic structure formation predicts that clusters of galaxies are formed via collisions and mergers of smaller groups and clusters. As shown by numerical simulations, merging plays a critical role in the cluster evolution. X-ray observations have provided many pieces of evidences for cluster mergers, through imaging infalling subclusters and disturbed, irregular morphologies of intracluster medium (ICM) (e.g., Forman \& Jones 1982). Furthermore, even in apparently relaxed clusters, discontinuous ICM structures so-called "cold fronts" are sometimes found from the Chandra observations. The cold fronts are interpreted as contact discontinuity caused by the subcluster collisions (Markevitch \& Vikhlinin 2007). Although the morphological data are accumulating, the physical states of the ICM, particularly the ionization equilibrium state and the kinematics, are yet to be clarified.

In the study of the dynamical evolution of clusters, we will focus on the cluster outer regions since they are expected to contain important information on the formation process. The

\footnotetext{
Last update: 2018 December 4
}

cluster outskirts are connected to the surrounding large-scale structure, where the gas is falling towards the cluster potential and possibly subject to shock heating. Once disturbed by the subcluster collisions, it should take long time for the gas to settle due to the low density and large spatial size. Therefore, the outskirts of merging clusters offer us opportunities to look into the gas in its transition to the thermal and ionization equilibriums.

Recent X-ray studies with Suzaku showed temperature structure of ICM to the virial radius $\left(r_{200}\right)$ for several relaxed clusters (George et al. 2008; Reiprich et al. 2009; Bautz et al. 2009; Hoshino et al. 2010; Simionescu et al. 2011). The results show a systematic drop of temperature by a factor of $\sim 3$ from the center to $r_{200}$. Also, the entropy shows a flattening or a small drop, after a monotonous increase with radius, around the outermost region. It has been interpreted as the deviation between electron and ion temperatures in this region. Numerical simulations for relaxed clusters also suggest that the outskirts of clusters are not in hydrostatic equilibrium (e.g. Burns et al. 2010).

Compared with the relaxed clusters, however, there is little information about the ICM properties in the outer regions of merging clusters. In those dynamically-young systems, the 
ICM are thought to be in the early stage of thermal relaxation. Thus, the X-ray observations of merging clusters and comparisons with the relaxed clusters will give us new, valuable information on the ICM properties at large radii and help us to draw the global picture of formation and evolution of clusters.

The surface brightness around the cluster virial radius is much lower than the central region (typically, by a factor of $10^{4}$ ). Thus, the detailed estimation of foreground and background emission as well as careful assessment of all the systematic errors is important in this kind of study. The XIS instrument on Suzaku has a low and stable background and the behavior of the non-X-ray background (NXB) is well known (Tawa et al. 2008; Koyama et al. 2007), which makes XIS the most suitable for the study of the cluster outer regions.

An additional science at cluster outer regions is the search for the warm-hot intergalactic medium (WHIM). The filamentary structure of the local universe has been probed mainly through galaxy distribution (e.g. Eisenstein et al. 2005). Its detailed structure would be directly observed by the WHIM, which has temperature of $10^{6}-10^{7} \mathrm{~K}$ and contains more than half of baryons in the local universe (e.g. Cen \& Ostriker 2006). Because of its extreme faintness, detailed observations of the WHIM will be the subject of future high-resolution X-ray studies. The gas density and chemical composition of the WHIM are still poorly known both theoretically and observationally. The cluster outskirts are the regions where the ICM is connected to the WHIM and will enable us to place observational constraints about the WHIM properties. As shown, by e.g., Takei et al. (2008), Suzaku XIS is able to set strong constraints about the redshifted oxygen emission.

Abell 2142 (A2142, $z=0.0909$ ) is a bright cluster of galaxies, having a high ICM temperature of $k T \approx 9 \mathrm{keV}$. This object is also known as the first cluster in which the cold fronts have been detected (Markevitch et al. 2000). The cold fronts in the south and the northwest are 0.7 (or $70 \mathrm{kpc}$ ) and 2.7 (or $270 \mathrm{kpc}$ ) off of the cluster center, respectively, and a sharp surface-brightness drop by a factor of about 2 is seen. Since the temperature and density distributions suggest that the pressure is constant across the cold front, it is considered as a contact discontinuity. The presence of these structures naturally indicates that A2142 is a merger and the subcluster infall seems to be occurring along the northwest-southeast direction. Actually the overall cluster emission is elongated in this direction. This is likely to coincide with the large-scale structure, and the matter density seems to be enhanced along the filament.

A2142 is also suitable for the search of emission lines from the WHIM. Given the cluster redshift of $\sim 0.1$, the OVII line (the rest-frame energy of $0.65 \mathrm{keV}$ ) is shifted to $0.57 \mathrm{keV}$ and then falls into a gap between the Galactic OVII line energy and the instrumental line features. Therefore, it makes it possible to distinguish the WHIM emission from the local one. In addition, because the redshift of A2142 is not too high, the oxygen line from the WHIM can be measured with good sensitivity. With these purposes, we have carried out Suzaku observations of the northwest offset regions of A2142.

In this paper, we will use $H_{0}=70 \mathrm{~km} \mathrm{~s}^{-1} \mathrm{Mpc}^{-1}, \Omega_{\mathrm{M}}=$ 0.27 and $\Omega_{\Lambda}=0.73$. This cosmology leads to $100.4 \mathrm{kpc}$ per arcmin at $z=0.0909$. The virial radius approximated by $r_{200}=$ $2.77 h_{70}^{-1}(\langle T\rangle / 10 \mathrm{keV})^{1 / 2} \mathrm{Mpc} / E(z)$, with $E(z)=\left(\Omega_{\mathrm{M}}(1+z)^{3}+1-\right.$
$\left.\Omega_{\mathrm{M}}\right)^{1 / 2}$ (Henry et al. 2009). For our cosmology and redshift, $r_{200}$ is $2.48 \mathrm{Mpc}(=24.8)$ with $k T=8.7 \mathrm{keV}$. In this paper, we adopted the solar abundance defined by Anders \& Grevesse (1989) and the Galactic $N_{\mathrm{H}}$ by Dickey \& Lockman (1990). The errors are in the $90 \%$ confidence for a single parameter.

\section{Observations and Data Reduction}

As shown in figure 1, we performed four pointing observations in 2007 with the XIS instrument around Abell 2142. The central pointing observation was performed in June, and the other three in September to October. The cold front feature indicates that there is an ongoing merger in the north-west to south-east direction. This suggests that matter would be falling in along this merger axis, possibly from a large-scale filament. We allocated the observed regions to be successively offset towards the north-west direction. The regions are designated as Center, Offset1, Offset2, and Offset 3 with the exposure times $51.4 \mathrm{ks}, 37 \mathrm{ks}, 58 \mathrm{ks}$, and $24 \mathrm{ks}$, respectively. The observation $\log$ is shown in table 1 . The outermost observation reaches twice the virial radius $(49.2 \sim 4.92 \mathrm{Mpc})$ from the cluster center, in which we planned to search for emission from the warmhot intergalactic medium (WHIM).

Three out of the four CCD chips were available in these observations: XIS0, XIS1 and XIS3. The XIS1 is a backilluminated chip with high sensitivity in the soft X-ray energy range. The effective area has been siginificantly reduced by the time of the observations due to the contamination building up on the IR/UV blocking filters. This effect along with its uncertainty are included in the effective area in our analysis. We used HEAsoft ver 6.9 and CALDB 2010-12-06. All the XIS sensors were in the normal clocking mode, and the spaced-row charge injection (SCI) was applied.

We extracted pulse-height spectra in 9 annular regions with

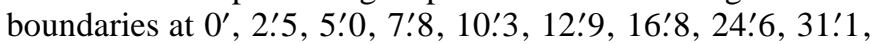
38.8 centered on $\left(15^{\mathrm{h}} 58^{\mathrm{m}} 16^{\mathrm{s}} 13,27^{\circ} 13^{\prime} 28^{\prime \prime}\right)$ from all the XIS events. We analyzed the spectra in the $0.5-10 \mathrm{keV}$ range for the FI detectors and $0.35-8 \mathrm{keV}$ for the BI detector. In all annuli, the calibration source positions were masked out using the calmask calibration database (CALDB) file.

\section{Background Analysis}

In the present study of the ICM emission in the cluster outskirts, correct estimation of the background is of utmost importance. As a standard practice, we assume three background components: non-X-ray background (NXB), cosmic $\mathrm{X}$-ray background (CXB) and Galactic emission. The Galactic emission component has a spatially variable spectrum. We estimated the Galactic background spectra using two Suzaku observation data, one was Offset 3 and another one was observation of TCrB (Observation ID = 401043010) which was located at 1 degree south of A2142. We will show each background component in this section.

\subsection{Cosmic X-ray Background}

Since the CXB consists of many extragalactic point sources, we tried to remove the sources as many as possible and then modeled the remaining emission with a power-law. We 
Table 1. Log of Suzaku observations of Abell 2142

\begin{tabular}{ccccc}
\hline \hline Position(Obs. ID) & Start & End & Exp. time (ks) $^{*}$ & Exp. time (ks) \\
\hline Center (801055010) & 2007 Jun 04 & 2007 Jun 05 & 51.4 & 45.2 \\
Offset1 (802030010) & 2007 Oct 04 & 2007 Oct 05 & 37.6 & 26.6 \\
Offset2 (802031010) & 2007 Sep 15 & 2007 Sep 17 & 57.6 & 41.3 \\
Offset3 (802032010) & 2007 Oct 29 & 2007 Oct 30 & 23.7 & 20.4 \\
T CrB (401043010) & 2006 Sep 06 & 2006 Sep 08 & 46.3 & 36.8 \\
\hline \hline *:COR2 >0 GV & $\dagger$ COR2 > 8 GV & & &
\end{tabular}

(a)

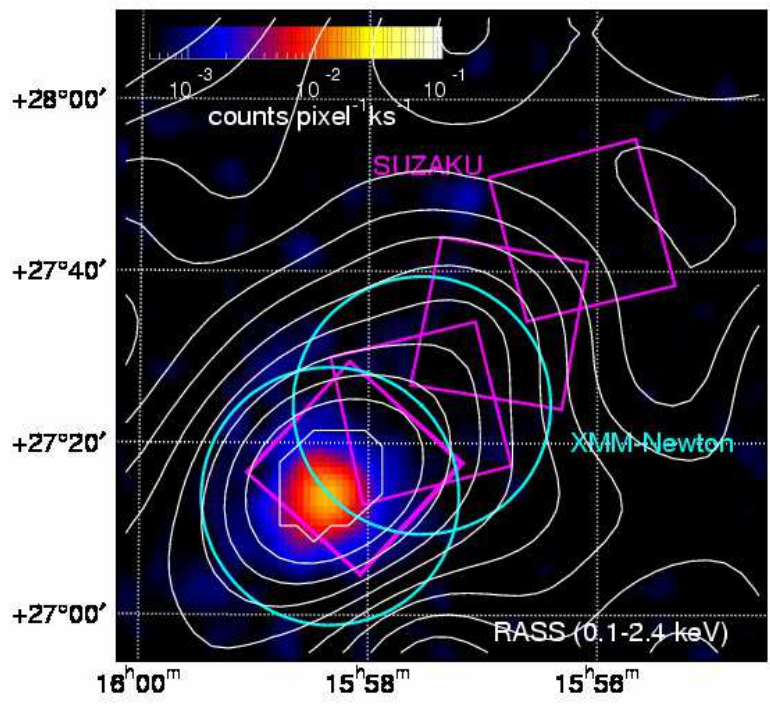

(b)

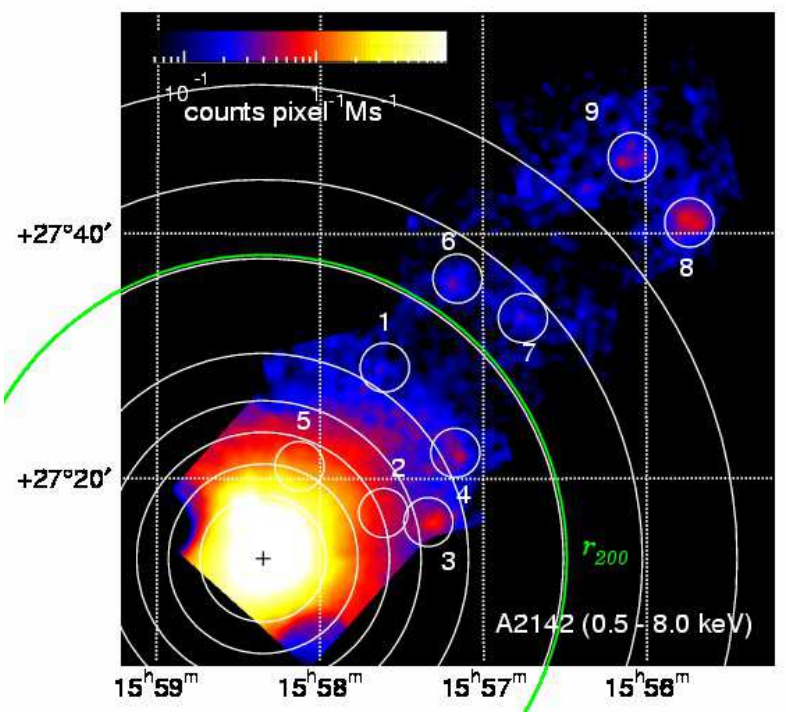

Fig. 1. (a):Rosat All Sky Survey image around A2142. Cyan circles show XMM-Newton FOV, and Magenta boxes show Suzaku Observations. White contours show galaxy distribution associated with the A2142 taken SDSS catalogue. (b):NXB subtracted Suzaku FI+BI image of A2142 in 0.5-8.0 keV band smoothed by a 2-dimensional gaussian with $\sigma=16 \mathrm{pixel}=17^{\prime \prime}$. The image is corrected for exposure time but not for vignetting. Large white circles indicate the regions used for spectrum analysis. Large green circle show the virial radius of A2142 ( $2.5 \mathrm{Mpc})$. Small white circles show the excluded point sources.

estimated the $\mathrm{CXB}$ surface brightness to be $5.97 \times 10^{-8}$ erg cm $\mathrm{cm}^{-2} \mathrm{~s}^{-1} \mathrm{sr}^{-1}$ after the source subtraction, based on ASCA GIS measurements (Kushino et al. 2002). We carefully subtracted point sources brighter than $8 \times 10^{-14} \mathrm{erg} \mathrm{cm}^{-2} \mathrm{~s}^{-1}$, while the flux limit of the point sources eliminated in Kushino et al. (2002) is $2 \times 10^{-13} \mathrm{erg} \mathrm{cm}^{-2} \mathrm{~s}^{-1}$. Our flux limit is thus sufficiently lower than Kushino et al. (2002). The details of pointsource subtraction are described in Appendix 1.

To estimate the amplitude of the CXB fluctuation, we scaled the fluctuation measured with Ginga (Hayashida et al. 1989) to our flux limit and field of view, following the analysis by Hoshino et al. (2010). The fluctuation amplitude scales as $\left(\Omega_{\mathrm{e}, \text { Suzaku }} / \Omega_{\mathrm{e}, \text { Ginga }}\right)^{-0.5}$, with $\Omega_{\mathrm{e}, \text { Suzaku }}$ and $\Omega_{\mathrm{e}, \text { Ginga }}$ the effective field of views (FOVs) of Suzaku and Ginga instruments, respectively. We show the resultant relative fluctuation $\sigma / I_{\mathrm{CXB}}$ for each annular region in table 3 , where $\sigma$ is the standard deviation of the CXB intensity $I_{\mathrm{CXB}}$.

\subsection{Non X-ray Background}

The non X-ray background (NXB) spectra were estimated from the database of Suzaku night-earth observations using the procedure of Tawa et al. (2008). We accumulated the data for the same detector area and the same distribution of COR2 as the A2142 observations, using an FTOOL xisnxbgen. The night-earth data cover 150 days before and after the period of A2142 observations. To increase the signal-to-noise ratio by keeping the NXB count rate low, we selected durations in which COR2 is $>8 \mathrm{GV}$. The systematic error due to the NXB uncertainty was estimated by varying the NXB intensity by $\pm 3 \%$ as in Tawa et al. (2008).

\subsection{Galactic Components}

To estimate the Galactic emission, we examined the spectra from two Suzaku observations: Offset3, which showed negligible ICM contribution, and TCrB at $1^{\circ}$ south of $\mathrm{A} 2142$. We employed ancillary response files (ARFs) for a spatially uniform source filling the FOV. A power-law is used to model the CXB in both spectra. The Galactic emission is represented as a two-temperature model consisting of an unabsorbed $\sim 0.1 \mathrm{keV}$ plasma (LHB; representing the local hot bubble and the solar wind charge exchange) and an absorbed $\sim 0.3 \mathrm{keV}$ plasma (MWH; representing the Milky Way halo): apec $_{1}+$ wabs $\times\left(\right.$ apec $_{2}+$ powerlaw $)$. The redshift and abundance of both the apec components were fixed at 0 and unity, respectively.

The best-fit parameters for the Offset 3 and $\mathrm{TCrB}$ regions are summarized in table 2. The temperatures of the LHB and the MWH are $0.09 \pm 0.02 \mathrm{keV}$ and $0.28 \pm 0.04 \mathrm{keV}$, respectively, 

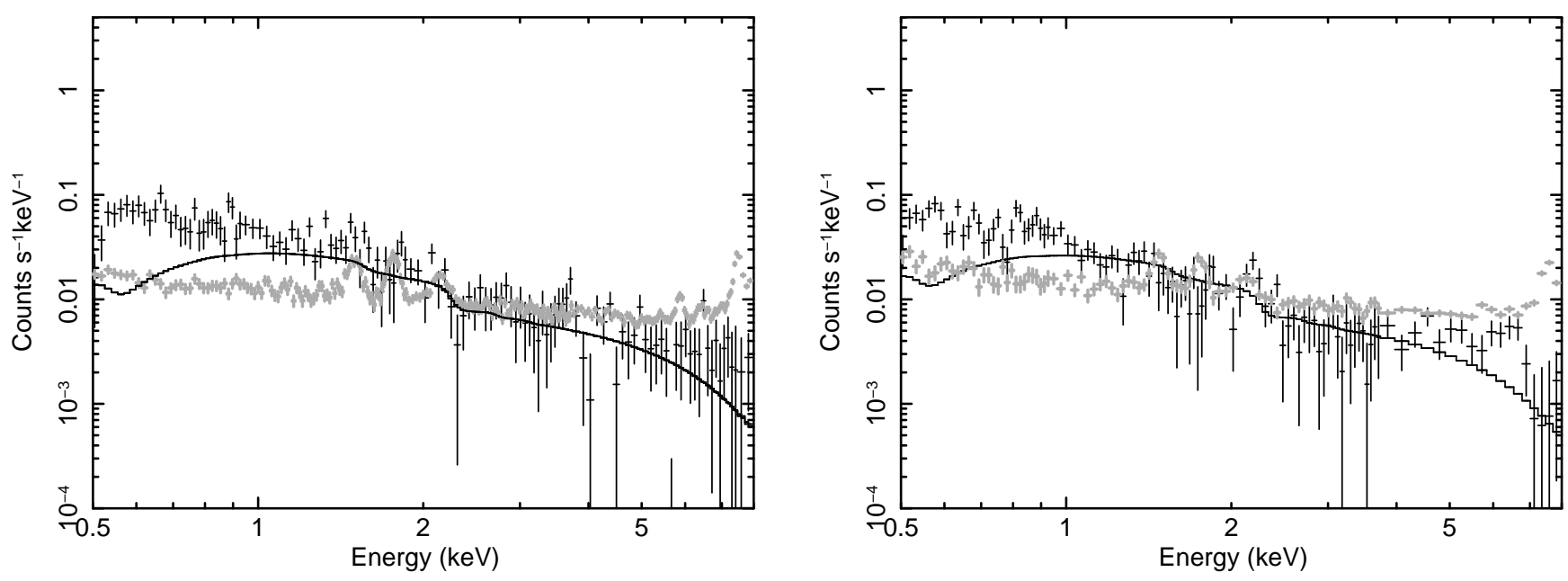

Fig. 2. NXB-subtracted XIS BI spectra (Black cross) of Offset3 (left) and TCrB(right), plotted with estimated CXB (black line) and NXB (gray cross) spectrum described in Sec 3.1, Sec 3.2 respectively.

for Offset 3 , and $0.09 \pm 0.02 \mathrm{keV}$ and $0.29 \pm 0.03 \mathrm{keV}$, respectively, for TCrB background. The temperatures and intensities are consistent with the typical Galactic emission. The observed fluxes in the two regions differ by $8 \%$, higher in Offset 3 . We assume that the difference of the two fluxes represents the typical uncertainty range and the systematic error of the Galactic emission.

\subsection{Background Fraction in Each Region}

Table 3 summarize the information in each annular region we analyzed. The columns indicate: the annular boundaries; $\Omega_{\mathrm{e}}$, the solid angle of observed areas; Coverage, the coverage fraction of each annulus, which is the ratio of $\Omega_{\mathrm{e}}$ to the total solid angle of the annulus; SOURCE_RATIO_REG, the fraction of the simulated cluster photons that fall in the region compared with the total photons generated in the entire simulated cluster; $\sigma / I_{\mathrm{CXB}}$, the CXB fluctuation due to unresolved point sources; OBS, the observed counts; the estimated counts for the three background components, i.e., NXB, CXB, and the Galactic emission; and the fraction of background photons given by $f_{\mathrm{BGD}} \equiv(\mathrm{NXB}+\mathrm{CXB}+$ Galactic $) / \mathrm{OBS}$.

The NXB counts are calculated from the night earth data. We simulated the spectra of the Galactic and CXB components, using xissim (Ishisaki et al. 2007) with the flux and spectral parameters given in the row of "A2142 OFFSET3 nominal" in table 2, assuming a uniform surface brightness that fills the XIS FOV. We plot the NXB and CXB BI spectra compared with the observed spectra in the background regions in figure 2 . These simulated spectra gave the counts shown in table 3 .

\section{Stray Light}

Stray light is photons entering from outside of the FOV. The Suzaku optics often show non-negligible stray light from nearby bright $X$-ray sources (Serlemitsos et al. 2007). The extended telescope point spread function also makes contamination of photons from a nearby sky. We estimated the contamination flux based on a ray tracing simulation xissim. The result of our simulation is shown in table 4 . For each observed annulus, we calculated the fraction originated from each sky area (i.e., annulus). We can see that the "on-source" fractions are naturally higher for the central pointing than for the other regions, since the on-source flux itself is higher. At the same time, $20-30 \%$ of the detected photons are due to the contamination, and they are mostly from adjacent regions. Therefore, the results indicate that even though the flux contamination is not negligible, the origin is limited to nearby regions. We proceeded to the spectral analysis without making correction for the stray light.

\section{Spectral Analysis}

\subsection{Spatial and Spectral Responses}

We need to calculate the spatial and spectral responses for the analysis of A2142 data. The response functions for extended sources are complicated because they depend on the surface brightness distribution of the source. They need to be calculated for each annular region. Monte Carlo simulator xissim incorporates the responses of the X-ray telescope and XIS instrument. The ARF generator using this simulator is called xissimarfgen (Ishisaki et al. 2007). We used version 2008-0405 of the simulator. The surface brightness distribution is one of the input parameters necessary to run xissim and xissimarfgen. Because of the extended PSF, the local efficiency is related with the relative flux among adjacent spatial elements. We used the $\beta$-model $\left(\beta=0.85, r_{c}=4.5\right)$ based on the ROSAT PSPC result as the input X-ray image (Henry \& Briel 1996).

We created ARFs assuming that the input image does not vary with energy. The effect of the contamination on the XIS IR/UV blocking filter is included in the ARFs based on the calibration in November 2006. The normalization of the ARF is defined such that the flux given as a result of the spectral fit is equal to the entire flux for a given spatial region.

\subsection{Spectral Fit}

We carried out spectral fitting to the pulse-height data of each annular region separately. The NXB component was subtracted before the fit, and the fitting model included the LHB, MWH, CXB and ICM components. The spectra from the BI and FI sensors were jointly fitted with the same model by min- 
Table 2. Best-fit values of background spectra fitting

\begin{tabular}{|c|c|c|c|c|c|c|}
\hline \multicolumn{7}{|c|}{ A2142 OFFSET3 } \\
\hline & Unabsorbed (keV) & norm $_{1}^{*}$ & $S_{1[0.4-10.0 \mathrm{keV}]}^{\dagger}$ & Absorbed (keV) & norm $_{2}^{*}$ & $S_{2[0.4-10.0 \mathrm{keV}]}^{\dagger}$ \\
\hline nominal & $0.090^{+0.020}$ & $4.90^{+4.76}$ & $0.59 \pm 0.02$ & $0.275^{+0.041}$ & $0.51^{+0.20}$ & $0.68 \pm 0.02$ \\
\hline CONTAMI $+10 \%$ & $0.090^{+0.0013}$ & $6.13^{+10.4}$ & $0.68 \pm 0.02$ & $0.279^{+0.03035}$ & $0.55^{+0.22}$ & $0.74 \pm 0.02$ \\
\hline CONTAMI-10\% & $0.095_{-0.021}^{+0.023}$ & $3.27_{-1.67}^{+2.23}$ & $0.53 \pm 0.02$ & $0.281_{-0.041}^{+0.0450}$ & $0.47_{-0.11}^{+0.18}$ & $0.63 \pm 0.02$ \\
\hline $\mathrm{NXB}+3 \%+\mathrm{MAXCXB}$ & $0.092_{-0.023}^{+0.021}$ & $4.01_{-2.19}^{+6.15}$ & $0.63 \pm 0.02$ & $0.266_{-0.038}^{+0.041}$ & $0.53_{-0.13}^{+0.11}$ & $0.72 \pm 0.02$ \\
\hline NXB-3\%+MINCXB & $0.094_{-0.019}^{+0.013}$ & $4.17_{-2.01}^{+3.20}$ & $0.59 \pm 0.02$ & $0.286_{-0.041}^{+0.038}$ & $0.47_{-0.11}^{+0.18}$ & $0.66 \pm 0.02$ \\
\hline \multicolumn{7}{|c|}{$\overline{\mathrm{TCrB}}$} \\
\hline & \multicolumn{3}{|c|}{ Unabsorbed plasma } & \multicolumn{3}{|c|}{ Absorbed plasma } \\
\hline & $\mathrm{kT}(\mathrm{keV})$ & norm $_{1}^{*}$ & $S_{1[0.4-10.0 \mathrm{keV}]}^{\dagger}$ & $\mathrm{kT}(\mathrm{keV})$ & norm $_{2}^{*}$ & $S_{2[0.4-10.0 \mathrm{keV}]}^{\dagger}$ \\
\hline nominal & $0.089_{-0.016}^{+0.010}$ & $3.85_{-132}^{+5.14}$ & $0.41 \pm 0.01$ & $0.292_{-0.026}^{+0.032}$ & $0.52_{-010}^{+0.09}$ & $0.72 \pm 0.02$ \\
\hline COMTAMI $+10 \%$ & 0.08 & $4.48^{+6.86}$ & $0.35 \pm 0.01$ & $0.291^{+0.0031}$ & $0.51^{+0.09}$ & $0.71 \pm 0.02$ \\
\hline CONTAMI-10\% & $0.087_{-0.013}^{+0.019}$ & $3.36_{-166}^{+3.82}$ & $0.42 \pm 0.01$ & $0.285_{-0.023}^{+0.052}$ & $0.45_{-0.12}^{+0.09}$ & $0.72 \pm 0.02$ \\
\hline $\mathrm{NXB}+3 \%+\mathrm{MAXCXB}$ & $0.086_{-0.015}^{+0.014}$ & $4.24_{-166}^{+6.47}$ & $0.44 \pm 0.01$ & $0.270^{+0.042}$ & $0.53^{+0.10}$ & $0.70 \pm 0.02$ \\
\hline NXB-3\%+MINCXB & $0.089_{-0.016}^{+0.009}$ & $3.95_{-1.27}^{+1.46}$ & $0.34 \pm 0.01$ & $0.293_{-0.025}^{+0.030}$ & $0.52_{-0.09}^{+0.09}$ & $0.60 \pm 0.02$ \\
\hline
\end{tabular}

*:Normalization of the apec component scaled with a factor $1 / 400 \pi$ assumed in the uniform-sky ARF calculation (circle radius $r=20^{\prime}$ ).

Norm $=\frac{1}{400 \pi} \int n_{\mathrm{e}} n_{\mathrm{H}} \mathrm{dV} /\left(4 \pi\left(1+z^{2}\right) D_{A}^{2}\right) \times 10^{-20} \mathrm{~cm}^{-5} \operatorname{arcmin}^{-2}$, where $D_{A}$ is the angular diameter distance to the source.

$\dagger: 10^{-6}$ photons $\mathrm{cm}^{-2} \mathrm{~s}^{-1} \operatorname{arcmin}^{-2}$. Energy band is $0.4-10.0 \mathrm{keV}$.

Table 3. Estimation of CXB fluctuation and Backgrand Count

\begin{tabular}{|c|c|c|c|c|c|c|c|c|c|}
\hline \multirow[t]{2}{*}{ Region } & \multirow{2}{*}{$\begin{array}{c}\Omega_{e}^{*} \\
\left(\operatorname{arcmin}^{2}\right) \\
\end{array}$} & \multirow{2}{*}{$\begin{array}{c}\text { Coverage }^{\dagger} \\
(\%)\end{array}$} & \multirow{2}{*}{$\begin{array}{l}\text { SOURCE } \\
\text { Ratio_Reg } \\
\end{array}$} & \multirow{2}{*}{$\begin{array}{c}\sigma / I_{C X B}^{\S} \\
(\%)\end{array}$} & \multicolumn{5}{|c|}{ FI Count $(0.5-10 \mathrm{keV})$} \\
\hline & & & & & OBS $\left(\times 10^{2}\right)$ & $\mathrm{NXB}\left(\times 10^{2}\right)$ & $\mathrm{CXB}\left(\times 10^{2}\right)$ & Galactic $\left(\times 10^{2}\right)$ & $f_{B G D}(\%)$ \\
\hline$\overline{0^{\prime}-2 ! 5}$ & $\overline{\overline{21.0}}$ & $\overline{100.0}$ & $\overline{\overline{27.0}}$ & $\overline{24.1}$ & $1701.8 \pm 4.1$ & $4.1 \pm 0.1$ & $3.9 \pm 0.9$ & $\overline{1.5 \pm 0.1}$ & $0.6 \pm 0.1$ \\
\hline $2 ! 5-5.0$ & 58.8 & 95.4 & 31.1 & 14.4 & $1131.2 \pm 3.4$ & $11.5 \pm 0.3$ & $9.9 \pm 1.4$ & $4.4 \pm 0.2$ & $2.3 \pm 0.4$ \\
\hline $5 ! 0-7 ! 8$ & 96.3 & 93.6 & 16.0 & 11.3 & $326.8 \pm 1.8$ & $10.8 \pm 0.3$ & $9.8 \pm 1.1$ & $3.8 \pm 0.2$ & $7.5 \pm 1.4$ \\
\hline $7 ! 8-10 ! 3$ & 126.6 & 86.2 & 7.7 & 9.8 & $122.0 \pm 1.1$ & $9.2 \pm 0.3$ & $7.2 \pm 0.7$ & $3.1 \pm 0.2$ & $16.1 \pm 3.1$ \\
\hline $10 ! 3-12 ! 9$ & 128.9 & 67.6 & 3.4 & 9.7 & $36.9 \pm 0.8$ & $6.1 \pm 0.2$ & $5.7 \pm 0.6$ & $2.5 \pm 0.2$ & $38.7 \pm 8.2$ \\
\hline $12 ! 9-16 ! 8$ & 101.8 & 42.5 & 1.1 & 10.3 & $31.8 \pm 0.6$ & $7.8 \pm 0.2$ & $7.1 \pm 0.8$ & $2.8 \pm 0.2$ & $55.7 \pm 11.2$ \\
\hline $16 ! 8-24 ! 6$ & 242.9 & 22.2 & 0.8 & 7.2 & $21.7 \pm 0.5$ & $8.5 \pm 0.3$ & $5.8 \pm 0.4$ & $2.4 \pm 0.2$ & $77.2 \pm 15.6$ \\
\hline $24 ! 6-31{ }^{\prime} \cdot 1$ & 191.6 & 15.8 & 0.2 & 8.1 & $38.9 \pm 0.6$ & $17.6 \pm 0.5$ & $16.8 \pm 1.4$ & $6.4 \pm 0.2$ & $104.9 \pm 17.1$ \\
\hline $31.1-38.8$ & 241.7 & 14.1 & 0.1 & 7.2 & $31.2 \pm 0.6$ & $16.5 \pm 0.5$ & $11.9 \pm 0.9$ & $5.2 \pm 0.2$ & $107.8 \pm 18.4$ \\
\hline \multirow[t]{2}{*}{ Region } & $\Omega_{e}^{*}$ & Coverage $^{\dagger}$ & Source & $\sigma / I_{C X B}^{\S}$ & \multicolumn{5}{|c|}{ BI Count $(0.5-8 \mathrm{keV})$} \\
\hline & $\left(\operatorname{arcmin}^{2}\right)$ & $(\%)$ & Ratio_Reg & $(\%)$ & OBS $\left(\times 10^{2}\right)$ & $\operatorname{NXB}\left(\times 10^{2}\right)$ & $\mathrm{CXB}\left(\times 10^{2}\right)$ & Galactic $\left(\times 10^{2}\right)$ & $f_{B G D}(\%)$ \\
\hline $0^{\prime}-2 ! 5$ & 21.0 & $\overline{100.0}$ & 27.0 & 24.1 & $1042.6 \pm 3.2$ & $\overline{3.0 \pm 0.1}$ & $1.9 \pm 0.5$ & $\overline{1.2 \pm 0.1}$ & $\overline{0.6 \pm 0.2}$ \\
\hline $2.5-5.0$ & 58.8 & 95.4 & 31.1 & 14.4 & $732.5 \pm 2.7$ & $8.2 \pm 0.2$ & $5.1 \pm 0.7$ & $3.8 \pm 0.2$ & $2.3 \pm 0.5$ \\
\hline $5 ! 0-7 ! 8$ & 95.8 & 92.5 & 15.5 & 11.5 & $245.4 \pm 1.6$ & $9.8 \pm 0.3$ & $6.1 \pm 0.7$ & $4.6 \pm 0.2$ & $8.4 \pm 1.6$ \\
\hline $7 ! \cdot 8-10 ! 3$ & 122.6 & 86.2 & 7.7 & 9.8 & $88.8 \pm 0.9$ & $7.8 \pm 0.2$ & $4.9 \pm 0.5$ & $3.2 \pm 0.2$ & $17.8 \pm 3.7$ \\
\hline $10 ! 3-12 ! 9$ & 125.9 & 64.3 & 3.0 & 9.4 & $30.5 \pm 0.7$ & $4.8 \pm 0.1$ & $3.3 \pm 0.3$ & $2.2 \pm 0.2$ & $33.8 \pm 7.8$ \\
\hline $12 ! 9-16 ! 8$ & 105.8 & 46.7 & 1.2 & 10.8 & $26.2 \pm 0.5$ & $6.0 \pm 0.2$ & $4.1 \pm 0.5$ & $2.8 \pm 0.2$ & $49.2 \pm 10.7$ \\
\hline $16 ! 8-24 ! 6$ & 233.9 & 20.8 & 0.7 & 8.2 & $18.6 \pm 0.4$ & $7.2 \pm 0.2$ & $3.6 \pm 0.3$ & $2.7 \pm 0.2$ & $72.8 \pm 15.6$ \\
\hline $24.6-311^{\prime} 1$ & 181.6 & 13.8 & 0.2 & 9.3 & $31.6 \pm 0.6$ & $14.6 \pm 0.4$ & $9.2 \pm 0.7$ & $6.2 \pm 0.2$ & $94.9 \pm 16.8$ \\
\hline $31.1-38.8$ & 225.7 & 11.1 & 0.1 & 7.6 & $24.6 \pm 0.5$ & $12.7 \pm 0.4$ & $6.8 \pm 0.5$ & $5.0 \pm 0.2$ & $99.3 \pm 18.4$ \\
\hline
\end{tabular}

imizing the total $\chi^{2}$ value. To increase the signal to noise ratio of A2142, we used energy ranges of $0.35-8 \mathrm{keV}$ for BI and $0.5-10 \mathrm{keV}$ for FI. The relative normalization between the two sensors was a free parameter in this fit to compensate for the cross-calibration errors. The photon index and normalization of the CXB, the temperatures and normalization of the LHB and MWH were fixed at the values in table 2. Metal abundances of the LHB and MWH components were set to be unity. The Galactic absorption column density was fixed at $N_{\mathrm{H}}=4.2 \times 10^{20} \mathrm{~cm}^{-2}$ (Dickey \& Lockman 1990). We checked the influence of an uncertainty in $N_{\mathrm{H}}$ by using another column density from Leiden/Argentine/Bonn (LAB) survey $\left(N_{\mathrm{H}}=3.8 \times 10^{20} \mathrm{~cm}^{-2}\right.$; Kalberla et al. 2005) to confirm that the two spectrum fits do not show significant difference. The fits were carried out with XSPEC ver12.4.0ao. In the central regions, free parameters were the temperature, normalization, metal abundance of the ICM component. In the outer regions, we fixed the metal abundance of the ICM at 0.2 , which is the 
Table 4. Stray light contamination for the central, OFFSET1 and OFFSET2 pointings

\begin{tabular}{|c|c|c|c|c|c|c|}
\hline \multicolumn{7}{|c|}{ CENTER } \\
\hline $\begin{array}{c}\text { Emission Weighted } \\
\text { Radius (arcmin) }\end{array}$ & $\begin{array}{l}\text { Detector } \\
/ \text { Sky }\end{array}$ & $0^{\prime}-2.5$ & $2.5-5.0$ & $5.0-7 ! 8$ & $7 ! .8-10 ! 3$ & $10 ! 3-12 ! 9$ \\
\hline $\bar{~} 1.1^{+1.5}$ & $\overline{00^{\prime}-2 ! 5}$ & 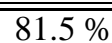 & $\overline{17.9 \%}$ & $0.5 \%$ & $\overline{0.1 \%}$ & $0.0 \%$ \\
\hline $3.4_{-09}^{+1.7}$ & $2.5-5 ! 0$ & $26.5 \%$ & $66.9 \%$ & $6.5 \%$ & $0.2 \%$ & $0.0 \%$ \\
\hline $5.9_{-07}^{+1.9}$ & $5 ! 0-7 ! 8$ & $5.3 \%$ & $33.8 \%$ & $56.0 \%$ & $4.8 \%$ & $0.1 \%$ \\
\hline $8.4^{-1.9}$ & $7 ! 8-10 ! 3$ & $4.3 \%$ & $8.1 \%$ & $31.4 \%$ & $51.9 \%$ & $4.3 \%$ \\
\hline $11.0_{-0.6}^{+1.9}$ & $10 ! 3-12 ! 9$ & $2.9 \%$ & $6.0 \%$ & $6.8 \%$ & $29.1 \%$ & $55.2 \%$ \\
\hline \multicolumn{7}{|c|}{ OFFSET1 and OFFSET2 } \\
\hline $\begin{array}{c}\text { Emission Weighted } \\
\text { Radius (arcmin) }\end{array}$ & $\begin{array}{l}\text { Detector } \\
\text { /Sky }\end{array}$ & $<12 ! 9$ & $12 ! 9-16 ! 8$ & $16 ! 8-24 ! 6$ & $24.6-31.1$ & $31.1-38.8$ \\
\hline- & $<12^{\prime} \cdot 9$ & $94.1 \%$ & $4.7 \%$ & $\overline{0.9 \%}$ & $0.2 \%$ & $\overline{0.0 \%}$ \\
\hline $14.0_{-1.1}^{+2.8}$ & $12: 9-16 ! 8$ & $21.2 \%$ & $61.7 \%$ & $15.8 \%$ & $1.2 \%$ & $0.0 \%$ \\
\hline $18.6_{-1.8}^{+5.9}$ & $16.8-24.6$ & $1.8 \%$ & $17.8 \%$ & $67.4 \%$ & $13.0 \%$ & $0.0 \%$ \\
\hline $26.3_{-1.8}^{+4.9}$ & $24.6-31 ! 1$ & $1.3 \%$ & $2.4 \%$ & $32.6 \%$ & $63.1 \%$ & $0.5 \%$ \\
\hline $32.5_{-1.4}^{+6.0}$ & $31{ }^{\prime} 1-38.8$ & $6.7 \%$ & $0.0 \%$ & $20.0 \%$ & $13.3 \%$ & $60.0 \%$ \\
\hline
\end{tabular}

lowest value observed in the outskirts of clusters (Fujita et al. 2008).

Figure 3 shows the results of the spectral fit for all the annular regions. The parameters and $\chi^{2}$ values are listed in tables 5 and 6 . We obtained fairly good fits for all the regions with reduced $\chi^{2}$ values less than 1.3.

\subsection{Temperature and Brightness Profiles}

Figure 4(a) shows the radial profile of ICM temperature, based on the result of the spectral fits. The inner 5 annular regions have the width of about 2.5 , and $20-40 \%$ of the detected flux comes from the adjacent sky regions. The maximum temperature within $7^{\prime}(700 \mathrm{kpc})$ from the cluster center is $\sim 9 \mathrm{keV}$, and it gradually decreases toward the outer region down to $\sim 4$ $\mathrm{keV}$ around the virial radius. There is a suggestion of weak emission with $k T \sim 1 \mathrm{keV}$ just outside of $r_{200}$. As shown in figure $3(\mathrm{~g})$ and $(\mathrm{h})$, this emission (in magenta) has the intensity much weaker than the other components throughout the energy range. If one takes into account the systematic error as shown in section 5.5, this component does not stay significant in the outermost region in figure $3(\mathrm{~h})$ and in table 5 and 6 . Therefore, we treat the $1 \mathrm{keV}$ emission in $31.1-38.8$ to be an upper limit.

Also, we check projection effect on temperature profiles. Based on Fujita et al. (2008), we re-fit each annulus with the following method.

- Fitting the ICM spectrum of the outermost region (24.'631.1 ) with a single ICM component.

- Fitting the next annulus, with a model of a combination of the ICM of this annulus and the overlapped emission from outer radius, i.e., the best fitting model of the outermost region normalized to account for the spherical projection.

- Proceeding to fitting of inner regions with a model of a combination of the ICM of this annulus and the overlapped emission from outer radius,

The results of this deprojection fitting do not show significant difference from the non-deprojection fitting. In sec 6 , we use the results of non-deprojection fitting method for discussion of ICM properties.

\subsection{Electron Density Profile}

The electron density profile was calculated from the normalization parameter of the Apec model, defined by

$$
N o r m=\frac{10^{-14}}{4 \pi D_{A}^{2}(1+z)^{2}} \int n_{e} n_{H} d V
$$

with the unit of $\mathrm{cm}^{-5}$, where $D_{A}(\mathrm{~cm})$ is the angular diameter distance to the source, $n_{e}$ and $n_{\mathrm{H}}\left(\mathrm{cm}^{-3}\right)$ are the number densities of electron and hydrogen, respectively. We note that the resultant normalization using an ARF generated by xissimarfgen needs the correction by a factor of SOURCE_RATIO_REG $/ \Omega_{e}$. See Ishisaki et al. 2007 Sec 5.3 for more information. Each annular region, projected in the sky, includes emission from different densities due to integration along the line of sight. We have de-convolved the electron density assuming spherical symmetry by successively calculating the value from the outermost regions. In this process, we assumed a constant temperature in each annular region. The uncertainty of the density due to this assumption is a few $\%$.

The resultant radial distribution of $n_{e}$ is shown in figure 4 (b), along with the $\beta$-model profile from ROSAT $\left(\beta=0.85, r_{c}=\right.$ 4.5). We obtained an upper limit of the electron density in the outermost region $\left(31^{\prime} 1-38 \prime^{\prime} 8\right)$ to be $<2.0 \times 10^{-5} \mathrm{~cm}^{-3}$. One can see that the $\beta$-model gives a fairly good approximation for the density profile, even though the temperature shows a large deviation from the isothermal case.

\subsection{Systematic Errors}

We have examined the effect of systematic errors on the derived spectral parameters. We considered 3 components for the systematic errors; namely, the NXB intensity with an error of $\pm 3 \%$ (Tawa et al. 2008), fluctuation of the CXB intensity, and contamination on the blocking filter. The CXB intensity was estimated in section 3.5 with the fluctuation shown in table 3 . We repeated all the spectral fits by fixing the CXB intensity at 
No. ]

(a) $0^{\prime}-2.5$

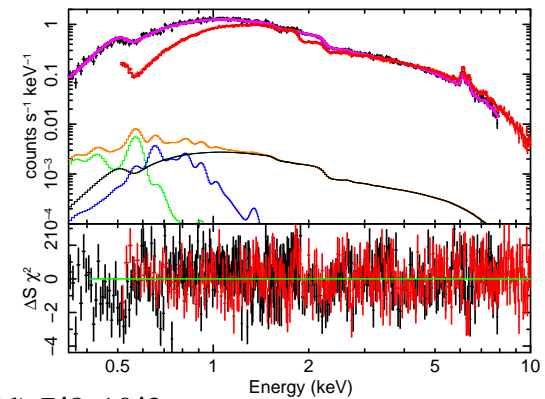

(d) $7 ! 8-10 ! 3$

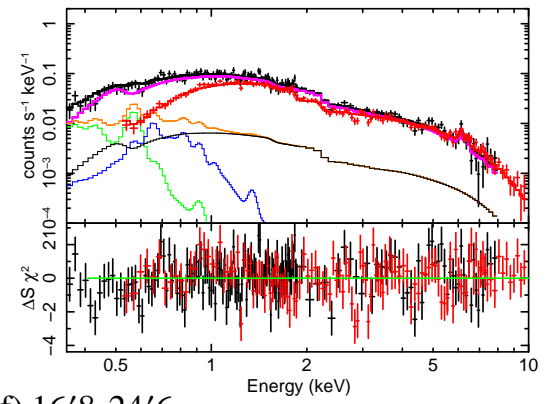

(f) $16.8-24.6$

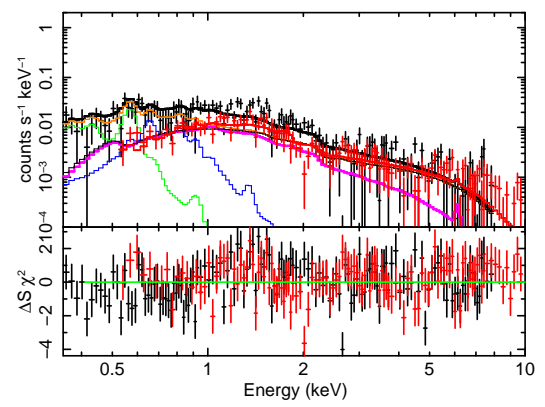

X-Ray Study of the Outer Region of Abell 2142 with Suzaku

(b) $2.5-5: 0$

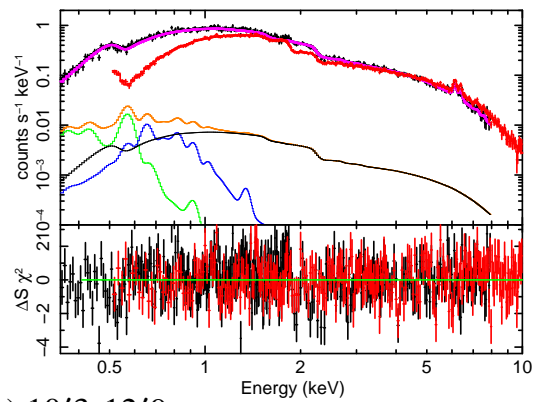

(e) 10!3-12!'

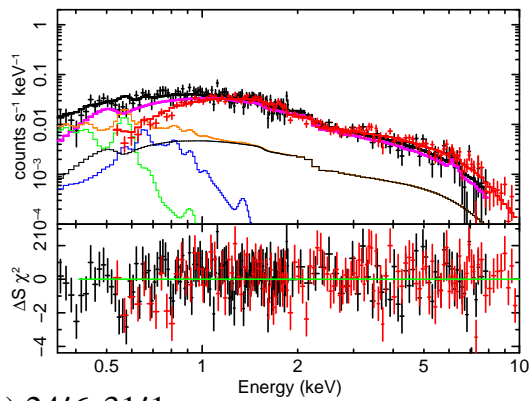

(g) 24.6-31!'

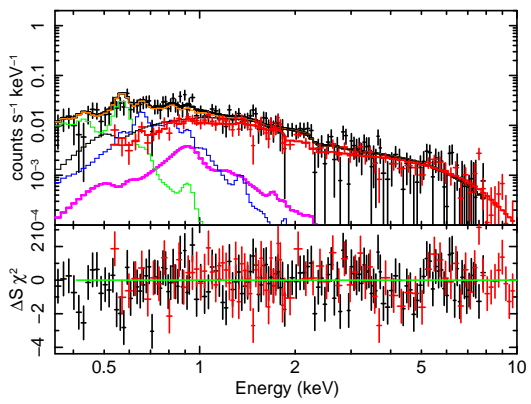

(c) $5 ! 0-7 ! 8$

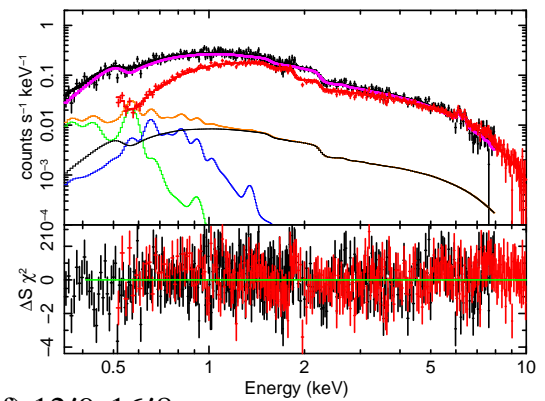

(f) $12 ! 9-16 ! 8$

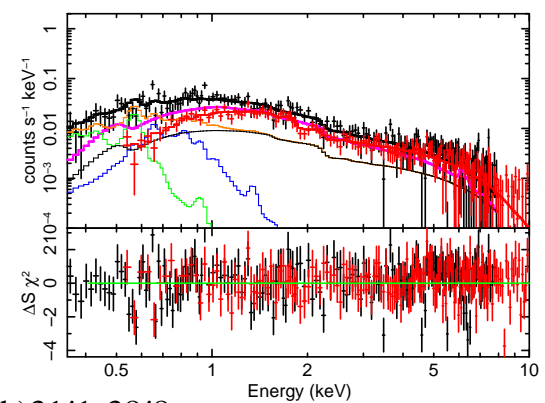

(h) $31 ! 1-38$ '.8

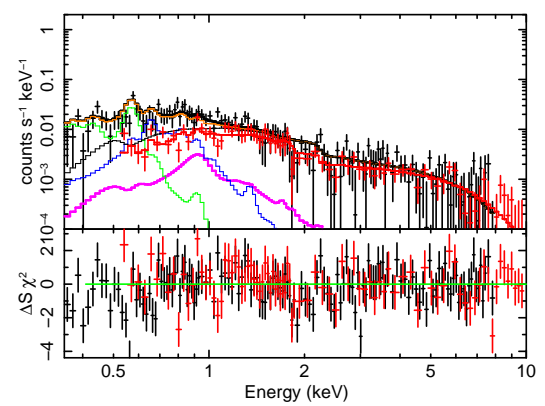

Fig. 3. NXB subtracted spectrum for each region. The XIS BI (Black) and FI (Red) spectra are fitted with ICM (wabs + apec), added to CXB+the galactic components (LHB, MWH) (apec+wabs(apec+powerlow)). The ICM components are shown in a magenta. The CXB components are shown in a black line, and the LHB and MWH emissions are indicated by green and blue lines, respectively.

the upper and lower boundary values, and the resultant parameters are listed in table 5. The top group shows the parameters with the nominal CXB intensity and contamination thickness. The parameters in CXBMAX group are for the higher CXB intensity, and those in CXBMIN are for the lower CXB intensity. The effects on the temperature is about $20 \%$ in the regions near the virial radius.

The other source of the systematic error is the uncertainty in the amount of contamination on the blocking filter of the XIS instrument. It showed a gradual increase during the observations of A2142, and the standard way is to include $10 \%$ error in the thickness of the contamination layer. The bottom 2 groups in tables 5 and 6 show the results. Since this error changes the detector response, all the regions are affected by the same amount. The change of temperature is 10-20\%.

The Galactic foreground emission is direction dependent even though the spectrum can be approximated by the sum of two thermal models with temperatures of about $0.1 \mathrm{keV}$ and 0.3 $\mathrm{keV}$. We looked into the results for two different backgrounds,
Offset 3 and TCrB regions, which can be treated as the systematic error due to the spatial variation of the Galactic emission. As seen in table 6, the effect becomes larger as the position goes farther from the cluster center. Near the virial radius, the difference in the ICM temperature is about $0.5 \mathrm{keV}$ yielding twice different temperatures.

\subsection{Search for WHIM Lines}

We searched for redshifted $\mathrm{O}$ lines in the spectra of all the annular regions, by adding gaussian lines in the spectral fit as shown in figure 5. The energies of the O VII and O VIII lines, assumed to have the same redshift of A2142 (0.0909), were fixed at $521 \mathrm{eV}$ and $598 \mathrm{eV}$, respectively. We also assumed the $\mathrm{O}$ abundance in the ICM to be 0 , which gives the highest or most conservative upper limits for the $\mathrm{O}$ lines. We obtained $2 \sigma$ upper limits of the $\mathrm{O}$ line intensity for all the regions as shown in table 7 . The average baryon density in the local universe is $1.77 \times 10^{-7}(1+z)^{3} \mathrm{~cm}^{-3}$ (Takei et al. 2008), or $2.3 \times 10^{-7} \mathrm{~cm}^{-3}$ at $z=0.0909$. Based on the observed upper limits of the line in- 
Table 5. Best-fit parameters of the ICM with Offset3 background

\begin{tabular}{|c|c|c|c|c|c|c|}
\hline Region & $\mathrm{k} T(\mathrm{keV})$ & $Z\left(Z_{\odot}\right)$ & norm* & $S_{04-10 \mathrm{keV}}^{\dagger}$ & $\mathrm{BI} / \mathrm{FI}$ & $\chi^{2} /$ d.o.f \\
\hline$\overline{0^{\prime}-2 ! 5}$ & $8.16_{-0.10}^{+0.10}$ & $0.33_{-0.02}^{+0.02}$ & $1.82 \pm 0.01 \times 10^{5}$ & $7.74 \pm 0.30 \times 10^{4}$ & $\overline{0.92}$ & $712 / 604$ \\
\hline 2. $5-5.0$ & $9.02_{-0.19}^{+0.19}$ & $0.28_{-0.02}^{+0.02}$ & $3.90 \pm 0.01 \times 10^{4}$ & $1.65 \pm 0.05 \times 10^{4}$ & 0.93 & $691 / 604$ \\
\hline $5 ! 0-7 ! 8$ & $\begin{array}{r}-0.199 \\
9.50_{-0.38}^{+0.38}\end{array}$ & $0.24_{-0.05}^{+0.05}$ & $7.50 \pm 0.01 \times 10^{3}$ & $3.33 \pm 0.05 \times 10^{3}$ & 1.03 & $681 / 604$ \\
\hline $7 ! 8-10 ! 3$ & $8.47_{-0.48}^{+0.65}$ & $0.31_{-0.09}^{+0.09}$ & $2.09 \pm 0.07 \times 10^{3}$ & $9.59 \pm 0.33 \times 10^{2}$ & 1.07 & $309 / 270$ \\
\hline $10^{\prime} 3-12.9$ & $7.79_{-0.79}^{+0.81}$ & $0.19_{-0.12}^{+0.13}$ & $8.30 \pm 0.32 \times 10^{2}$ & $3.90 \pm 0.33 \times 10^{2}$ & 1.19 & $338 / 270$ \\
\hline $12 ! 9-16 ! 8$ & $6.14_{-0.95}^{+1.30}$ & 0.2 (fix) & $6.70 \pm 0.40 \times 10^{2}$ & $2.67 \pm 0.22 \times 10^{2}$ & 0.85 & $381 / 358$ \\
\hline $16^{\prime} 8-24^{\prime} 6$ & $3.42_{-1.22}^{+2.14}$ & 0.2 (fix) & $1.35 \pm 0.22 \times 10^{2}$ & $5.18 \pm 0.22 \times 10^{1}$ & 0.92 & $272 / 217$ \\
\hline $24 .^{\prime} 6-31{ }^{\prime} 1$ & $1.01_{-029}^{+0.44}$ & 0.2 (fix) & $2.00 \pm 0.11 \times 10^{1}$ & $6.40 \pm 0.20$ & 0.94 & $238 / 223$ \\
\hline $31^{\prime} 1-38^{\prime} 8$ & $1.08_{-0.37}^{+0.28}$ & 0.2 (fix) & $1.00 \pm 0.50$ & $2.75 \pm 0.20$ & 0.88 & $299 / 223$ \\
\hline \multicolumn{7}{|c|}{ CXBMAX } \\
\hline $00^{\prime}-2 ! 5$ & $8.16_{-0.10}^{+0.10}$ & $0.33_{-0.02}^{+0.02}$ & $1.76 \pm 0.01 \times 10^{5}$ & $7.52 \pm 0.30 \times 10^{4}$ & 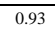 & $7112 / 604$ \\
\hline 2. $5-5.0$ & $8.99_{-0.19}^{+0: 19}$ & $0.28_{-0.02}^{+0.02}$ & $3.80 \pm 0.05 \times 10^{4}$ & $1.60 \pm 0.06 \times 10^{4}$ & 0.93 & $690 / 604$ \\
\hline $5 ! 0-7 ! 8$ & $9.39_{-0.38}^{+0.38}$ & $0.23_{-0.05}^{+0.05}$ & $7.37 \pm 0.13 \times 10^{3}$ & $3.23 \pm 0.05 \times 10^{3}$ & 1.03 & $675 / 604$ \\
\hline $7 ! 8-10 ! 3$ & $8.36_{-0.48}^{+0.60}$ & $0.30_{-0.09}^{+0.09}$ & $2.05 \pm 0.06 \times 10^{3}$ & $9.35 \pm 0.32 \times 10^{2}$ & 1.07 & $301 / 270$ \\
\hline $10 ! 3-12 ! 9$ & $\begin{array}{r}-0.48 \\
7.65_{-0.79}^{+0.81}\end{array}$ & $0.18_{-0.12}^{+0.12}$ & $8.18 \pm 0.32 \times 10^{2}$ & $3.82 \pm 0.32 \times 10^{2}$ & 1.18 & $337 / 270$ \\
\hline $12 !^{\prime} 9-16^{\prime} 8$ & $6.26_{-1.00}^{+1.40}$ & 0.2 (fix) & $6.96 \pm 0.48 \times 10^{2}$ & $2.75 \pm 0.23 \times 10^{2}$ & 0.84 & $376 / 358$ \\
\hline $16^{\prime} 8-24^{\prime} 6$ & $3.96_{-1.27}^{+2.66}$ & 0.2 (fix) & $1.45 \pm 0.24 \times 10^{2}$ & $5.64 \pm 0.29 \times 10^{1}$ & 0.90 & $273 / 217$ \\
\hline $244^{\prime} 6-31^{\prime} 1$ & $1.06_{-0.41}^{+0.49}$ & 0.2 (fix) & $2.16 \pm 1.21 \times 10^{1}$ & $7.55 \pm 0.40$ & 0.89 & $231 / 223$ \\
\hline $311^{\prime} 1-38^{\prime} .8$ & $1.02_{-0.47}^{+0.43}$ & 0.2 (fix) & $6.80 \pm 5.80$ & $2.35 \pm 0.03$ & 0.82 & $288 / 223$ \\
\hline \multicolumn{7}{|c|}{ CXBMIN } \\
\hline $0^{\prime}-2 ! 5$ & $8.17_{-0.10}^{+0.10}$ & $0.33_{-0.02}^{+0.02}$ & $1.87 \pm 0.01 \times 10^{5}$ & $7.98 \pm 0.31 \times 10^{4}$ & 0.92 & $713 / 604$ \\
\hline 2. $5-5.0$ & $\begin{array}{r}9.07_{-0.19}^{+0.19} \\
\text { (1) }\end{array}$ & $0.28_{-0.02}^{+0.02}$ & $4.04 \pm 0.05 \times 10^{4}$ & $1.70 \pm 0.06 \times 10^{4}$ & 0.93 & $693 / 604$ \\
\hline $5.0-7{ }^{\prime} 8$ & $\begin{array}{r}-0.49 \\
9.65_{-0.38}^{+0.38}\end{array}$ & $0.24_{-0.05}^{+0.05}$ & $7.75 \pm 0.13 \times 10^{3}$ & $3.44 \pm 0.05 \times 10^{3}$ & 1.03 & $687 / 604$ \\
\hline $7 ! 9-10 ! 3$ & $8.82_{-0.56}^{+0.70}$ & $\begin{array}{l}0.31_{-0.09}^{+0.09} \\
\text { - }\end{array}$ & $2.19 \pm 0.06 \times 10^{3}$ & $1.00 \pm 0.03 \times 10^{3}$ & 1.07 & $311 / 270$ \\
\hline $10 ! 3-12 ! 9$ & $8.34_{-0.78}^{+0.93}$ & $0.19_{-0.13}^{+0.13}$ & $8.82 \pm 0.30 \times 10^{2}$ & $4.14 \pm 0.34 \times 10^{2}$ & 1.18 & $339 / 270$ \\
\hline $12 ! 9-16 ! 8$ & $6.29_{-0.94}^{+1.32}$ & 0.2 (fix) & $7.69 \pm 0.48 \times 10^{2}$ & $2.33 \pm 1.81 \times 10^{2}$ & 1.19 & $431 / 358$ \\
\hline $16 ! 8-24 !^{\prime} 6$ & $3.38_{-0.62}^{+0.84}$ & 0.2 (fix) & $3.47 \pm 0.25 \times 10^{2}$ & $7.35 \pm 0.60 \times 10^{1}$ & 1.26 & $255 / 217$ \\
\hline $244^{\prime} 6-31^{\prime} 1$ & $1.13_{-0.30}^{+1.40}$ & 0.2 (fix) & $1.97 \pm 1.20 \times 10^{1}$ & $7.05 \pm 0.20$ & 0.97 & $242 / 223$ \\
\hline $31 !^{\prime} 1-38 ! 8$ & $1.08_{-0.30}^{+0.43}$ & 0.2 (fix) & $7.20 \pm 5.20$ & $2.55 \pm 0.02$ & 0.94 & $304 / 223$ \\
\hline \multicolumn{7}{|c|}{ CONTAMI $10 \%$ add } \\
\hline $0^{\prime}-2 ! 5$ & $7.85_{-0.10}^{+0.10}$ & $0.33_{-0.02}^{+0.02}$ & $1.86 \pm 0.01 \times 10^{5}$ & $7.86 \pm 0.03 \times 10^{4}$ & 0.92 & $734 / 604$ \\
\hline 2. $5-5.0$ & $8.57_{-0.14}^{+0: 19}$ & $0.28_{-0.02}^{+0.02}$ & $3.94 \pm 0.05 \times 10^{4}$ & $1.67 \pm 0.06 \times 10^{4}$ & 0.93 & $710 / 604$ \\
\hline $5.0-7 ?^{\prime} 8$ & $9.03_{-0.37}^{+0.38}$ & $0.23_{-0.04}^{+0.04}$ & $7.62 \pm 0.13 \times 10^{3}$ & $3.73 \pm 0.05 \times 10^{3}$ & 1.03 & $685 / 604$ \\
\hline $7 !^{\prime} 8-10 ! 3$ & $8.18_{-0.47}^{+0.50}$ & $0.30_{-0.08}^{+0.08}$ & $2.15 \pm 0.06 \times 10^{3}$ & $9.74 \pm 0.29 \times 10^{2}$ & 1.06 & $300 / 270$ \\
\hline $10 ! 3-12,9$ & $7.57_{-0.77}^{+0.80}$ & $0.18_{-0.12}^{+0.12}$ & $8.50 \pm 0.03 \times 10^{2}$ & $3.97 \pm 0.30 \times 10^{2}$ & 1.18 & $325 / 270$ \\
\hline $12^{\prime} 9-16^{\prime} 8$ & $5.72_{-0.83}^{+1.06}$ & 0.2 (fix) & $6.96 \pm 0.48 \times 10^{2}$ & $2.73 \pm 0.23 \times 10^{2}$ & 0.84 & $376 / 358$ \\
\hline $16^{\prime} 8-24^{\prime} 6$ & $3.22_{-0.94}^{+1.69}$ & 0.2 (fix) & $1.45 \pm 0.24 \times 10^{1}$ & $5.48 \pm 0.27 \times 10^{1}$ & 0.91 & $264 / 217$ \\
\hline $24{ }^{\prime} 6-31^{\prime} 11$ & $1.08_{-0.46}^{+2.01}$ & 0.2 (fix) & $1.08 \pm 0.90 \times 10^{1}$ & $4.00 \pm 1.00 \times 10^{0}$ & 0.95 & $255 / 223$ \\
\hline $31^{\prime} 1-38^{\prime} .8$ & -0 & - & - & $=$ & $1: 0.88$ & $319 / 223$ \\
\hline \multicolumn{7}{|c|}{ CONTAMI $10 \%$ red } \\
\hline $0^{\prime}-2 ! 5$ & $8.27_{-0.10}^{+0.10}$ & $0.34_{-0.02}^{+0.02}$ & $1.81 \pm 0.01 \times 10^{5}$ & $7.71 \pm 0.03 \times 10^{4}$ & 0.93 & $764 / 604$ \\
\hline 2. $5-5.0$ & $9.22_{-0.19}^{+0.19}$ & $0.28_{-0.02}^{+0.02}$ & $3.89 \pm 0.05 \times 10^{4}$ & $1.64 \pm 0.05 \times 10^{4}$ & 0.94 & $747 / 604$ \\
\hline $5 \cdot 0-7 ?^{\prime} 8$ & $9.81_{-0.38}^{+0.38}$ & $0.24_{-0.05}^{+0.05}$ & $7.50 \pm 0.13 \times 10^{3}$ & $3.31 \pm 0.06 \times 10^{3}$ & 1.04 & $706 / 604$ \\
\hline $7 ! 8-10 ! 3$ & $8.80_{-0.54}^{+0.71}$ & $0.31_{-0.09}^{+0.09}$ & $2.09 \pm 0.06 \times 10^{3}$ & $9.54 \pm 0.34 \times 10^{2}$ & 1.07 & $322 / 270$ \\
\hline $10^{\prime} 3-12.9$ & $8.39_{-0.81}^{+0.96}$ & $0.18_{-0.13}^{+0.14}$ & $8.18 \pm 0.32 \times 10^{2}$ & $3.86 \pm 0.34 \times 10^{2}$ & 1.19 & $357 / 270$ \\
\hline $12 ! 9-16 ! 8$ & $6.60_{-1.08}^{+1.57}$ & 0.2 (fix) & $6.56 \pm 0.40 \times 10^{2}$ & $2.60 \pm 0.20 \times 10^{2}$ & 0.85 & $392 / 358$ \\
\hline $16^{\prime} 8-24 ! 6$ & $4.15_{-1}^{+3.52}$ & 0.2 (fix) & $1.24 \pm 0.24 \times 10^{2}$ & $4.89 \pm 0.18 \times 10^{1}$ & 0.93 & $286 / 217$ \\
\hline $24{ }^{\prime} 6-31^{\prime} 11$ & $0.99_{-0.26}^{+0.37}$ & 0.2 (fix) & $1.91 \pm 1.10 \times 10^{1}$ & $7.00 \pm 0.20$ & 0.94 & $226 / 223$ \\
\hline $31 ! 1-38 ! 8$ & $1.06_{-0.34}^{+0.27}$ & 0.2 (fix) & $9.10 \pm 5.40$ & $3.15 \pm 0.03$ & 0.87 & $283 / 223$ \\
\hline
\end{tabular}

Norm $=\frac{\text { SOURCERATIO_REG }}{\Omega_{e}} \int \mathrm{n}_{\mathrm{e}} \mathrm{n}_{\mathrm{H}} \mathrm{dV} /\left(4 \pi\left(1+\mathrm{z}^{2}\right) \mathrm{D}_{\mathrm{A}}^{2}\right) \times 10^{-22} \mathrm{~cm}^{-5} \operatorname{arcmin}^{-2}$,

where $D_{A}$ is the angular diameter distance to the source.

t: $10^{-8}$ photonscm ${ }^{-2} \mathrm{~s}^{-1} \operatorname{arcmin}^{-2}$. Energy band is $0.4-10.0 \mathrm{keV}$.

Surface brightness of the apec component scaled with a factor SOURCE-RATIO-REG $\Omega_{e}$ from table 3.

tensities, we derived upper limits of the over-density $(\delta)$ of the gas assuming the line-of-sight depth of $2 \mathrm{Mpc}$ and the temperature of $2 \times 10^{6} \mathrm{~K}$. These assumptions are the same as in Takei et al. (2008). The results are summarized in table 7 for the two different Galactic backgrounds separately. We note that the lowest upper limits, with overdensity less than 280 (assuming background of Offset3) or 380 (assuming background of TCrB) is obtained for the outermost region $(r=31.0-38.7)$. We also derived upper limits of the overdensity from the measured electron density. In sec 5.4, we showed the $1 \sigma$ upper-limit of the electron density to be $<2.0 \times 10^{-5} \mathrm{~cm}^{-3}$ in the outermost region. In the range of $2 \sigma$, the upper limit of the electron density is $<2.6 \times 10^{-5} \mathrm{~cm}^{-3}$, corresponding to an overdensity $<113$.
Table 6. Best-fit parameters of the ICM with TCrB background

\begin{tabular}{|c|c|c|c|c|c|c|}
\hline Region & $\mathrm{k} T(\mathrm{keV})$ & $Z\left(Z_{\odot}\right)$ & norm* & $S_{04-10 \mathrm{keV}}^{\dagger}$ & $\mathrm{BI} / \mathrm{FI}$ & $\chi^{2} /$ d.o.f \\
\hline $0^{\prime}-2.5$ & $8.16_{-010}^{+0.10}$ & $0.33_{-0.02}^{+0.02}$ & $1.82 \pm 0.01 \times 10^{5}$ & $7.74 \pm 0.03 \times 10^{4}$ & 0.92 & $711 / 604$ \\
\hline 2! $5-5.0$ & $9.02_{-0.19}^{+0.19}$ & $0.28_{-0.02}^{+0.02}$ & $3.89 \pm 0.05 \times 10^{4}$ & $1.65 \pm 0.06 \times 10^{4}$ & 0.93 & $689 / 604$ \\
\hline $5 ! 0-7 ! 8$ & $9.47^{+0.38}$ & $0.24^{-0.05}$ & $7.50 \pm 0.13 \times 10^{3}$ & $3.33 \pm 0.04 \times 10^{3}$ & 1.03 & $674 / 604$ \\
\hline $7 ! 8-10 ! 3$ & $\begin{array}{r}-0.60 \\
8.47_{-0.48}^{+0.66}\end{array}$ & $\begin{array}{r}0.01 .09 \\
0.01_{-0.09}^{+0.09}\end{array}$ & $2.12 \pm 0.07 \times 10^{3}$ & $9.63 \pm 0.29 \times 10^{2}$ & 1.06 & $298 / 270$ \\
\hline $10 ! 3-12 ! 9$ & $7.84_{-0.79}^{+0.82}$ & $0.19_{-0.12}^{+0.13}$ & $8.39 \pm 0.32 \times 10^{2}$ & $3.94 \pm 0.31 \times 10^{2}$ & 1.17 & $323 / 270$ \\
\hline $12.9-16^{\prime} 8$ & $6.02_{-0.91}^{+1.21}$ & 0.2 (fix) & $6.88 \pm 0.48 \times 10^{2}$ & $2.70 \pm 0.24 \times 10^{2}$ & 0.84 & $373 / 358$ \\
\hline $16 ! 8-24{ }^{\prime} 6$ & $3.62_{-1.14}^{+1.916}$ & 0.2 (fix) & $1.43 \pm 0.24 \times 10^{2}$ & $5.47 \pm 3.05 \times 10^{1}$ & 0.89 & $264 / 217$ \\
\hline $24 ! 6-31^{\prime} 11$ & $1.07_{-0.31}^{+0.55}$ & 0.2 (fix) & $1.91 \pm 1.21 \times 10^{1}$ & $6.75 \pm 0.25$ & 0.97 & $228 / 223$ \\
\hline $31^{\prime} 1-38^{\prime} 8$ & $\begin{array}{l}1.07_{-0.30}^{+0.35} \\
\end{array}$ & 0.2 (fix) & $9.11 \pm 6.07$ & $3.15 \pm 0.25$ & 0.87 & $275 / 223$ \\
\hline \multicolumn{7}{|c|}{ CXBMAX } \\
\hline $0^{\prime}-2 ! 5$ & $8.16_{-0.10}^{+0.10}$ & $0.33_{-0.02}^{+0.02}$ & $1.82 \pm 0.01 \times 10^{5}$ & $\overline{7.73 \pm 0.03 \times 10^{4}}$ & 0.92 & $711 / 604$ \\
\hline 2! $5-5.0$ & $9.01_{-0.19}^{+0.19}$ & $0.28_{-0.02}^{+0.02}$ & $3.89 \pm 0.05 \times 10^{4}$ & $1.65 \pm 0.06 \times 10^{4}$ & 0.93 & $689 / 604$ \\
\hline $5 ! 0-7 ! 8$ & $9.46^{+0.38}$ & $0.24^{-0.05}$ & $7.50 \pm 0.13 \times 10^{3}$ & $3.31 \pm 0.05 \times 10^{3}$ & 1.03 & $674 / 604$ \\
\hline $7 ! 8-10 ! 3$ & $8.46^{+0.686}$ & $0.31^{-0.09}$ & $2.09 \pm 0.76 \times 10^{3}$ & $9.54 \pm 0.30 \times 10^{2}$ & 1.06 & $299 / 270$ \\
\hline $10^{\prime} 3-12^{\prime} 9$ & $7.81_{-0.80}^{+0.84}$ & $0.19_{-0.13}^{+0.139}$ & $8.18 \pm 0.32 \times 10^{2}$ & $3.86 \pm 0.31 \times 10^{2}$ & 1.18 & $324 / 270$ \\
\hline $12 !^{\prime} 9-16^{\prime} 8$ & $\begin{array}{l}5.87_{-0.90}^{+1.20} \\
-\end{array}$ & 0.2 (fix) & $6.56 \pm 0.40 \times 10^{2}$ & $2.57 \pm 0.22 \times 10^{2}$ & 0.84 & $373 / 358$ \\
\hline $16 ! 8-24{ }^{\prime} 6$ & $3.21_{-1.03}^{+1.93}$ & 0.2 (fix) & $1.28 \pm 0.24 \times 10^{1}$ & $4.81 \pm 0.22 \times 10^{1}$ & 0.91 & $267 / 217$ \\
\hline $24 ! 6-31 ! \cdot 1$ & $2.10_{-0.32}^{+0.56}$ & 0.2 (fix) & $7.00 \pm 1.55$ & $2.55 \pm 0.15$ & 0.91 & $236 / 223$ \\
\hline $31^{\prime} 1-38 ! 8$ & & - & - & - & 0.87 & $287 / 223$ \\
\hline \multicolumn{7}{|c|}{ CXBMIN } \\
\hline $0^{\prime}-2 ! 5$ & $8.16_{-010}^{+0.10}$ & $0.33_{-0.02}^{+0.02}$ & $1.82 \pm 0.01 \times 10^{5}$ & $7.74 \pm 0.30 \times 10^{4}$ & 0.92 & $712 / 604$ \\
\hline $2 ! 5-5 \prime^{\prime} 0$ & $9.03+0.19$ & $0.28^{+0.02}$ & $3.89 \pm 0.05 \times 10^{4}$ & $1.65 \pm 0.06 \times 10^{4}$ & 0.93 & $689 / 604$ \\
\hline $5 ! 0-7 ! 8$ & $9.51_{-0.38}^{+0.38}$ & $0.24_{-0.05}^{+0.05}$ & $7.62 \pm 0.13 \times 10^{3}$ & $3.34 \pm 0.51 \times 10^{3}$ & 1.03 & $675 / 604$ \\
\hline $7 ! 8-10 ! 3$ & $8.53_{-0.47}^{+0.68}$ & $0.31_{-0.09}^{-0.09}$ & $2.12 \pm 0.07 \times 10^{3}$ & $9.69 \pm 0.36 \times 10^{2}$ & 1.07 & $299 / 270$ \\
\hline $10^{\prime} 3-12^{\prime} 9$ & $7.95_{-0.78}^{+0.84}$ & $0.19_{-0.12}^{+0.93}$ & $8.50 \pm 0.32 \times 10^{2}$ & $3.98 \pm 0.32 \times 10^{2}$ & 1.18 & $324 / 270$ \\
\hline $12^{\prime} 9-16^{\prime} 8$ & $6.57_{-1.12}^{+1.52}$ & 0.2 (fix) & $7.28 \pm 0.48 \times 10^{2}$ & $2.79 \pm 0.24 \times 10^{2}$ & 0.84 & $372 / 358$ \\
\hline $16^{\prime} 8-24{ }^{\prime} 6$ & $3.96_{-1.18}^{+2.28}$ & 0.2 (fix) & $1.47 \pm 0.22 \times 10^{2}$ & $5.71 \pm 0.28 \times 10^{1}$ & 0.91 & $268 / 217$ \\
\hline $24.6-311^{\prime} 1$ & $1.15_{-0.29}^{+1.56}$ & 0.2 (fix) & $2.23 \pm 0.14 \times 10^{1}$ & $8.10 \pm 0.60$ & 0.99 & $222 / 223$ \\
\hline $31 ! 1-38{ }^{\prime} 8$ & $1.09_{-0.24}^{+0.43}$ & 0.2 (fix) & $9.57 \pm 6.30$ & $3.35 \pm 0.15$ & 0.92 & $273 / 223$ \\
\hline \multicolumn{7}{|c|}{ CONTAMI $10 \%$ ADD } \\
\hline $00^{\prime}-2 ! 5$ & $7.86^{+0.10}$ & $0.33^{+0.02}$ & $1.86 \pm 0.01 \times 10^{3}$ & $7.86 \pm 0.32 \times 10^{4}$ & 0.92 & $734 / 604$ \\
\hline $2 ! 5-5{ }^{\prime} 0$ & $8.57^{+0.20}$ & $0.28^{+0.02}$ & $3.94 \pm 0.05 \times 10^{4}$ & $1.67 \pm 0.06 \times 10^{4}$ & 0.93 & $710 / 604$ \\
\hline $5 ! 0-7 ! 8$ & $9.05_{-0.37}^{+0.38}$ & $0.23_{-0.04}^{+0.84}$ & $7.62 \pm 0.13 \times 10^{3}$ & $3.37 \pm 0.05 \times 10^{3}$ & 1.03 & $684 / 604$ \\
\hline $7 ! 8-10 ! 3$ & $8.21_{-0.47}^{+0.52}$ & $\begin{array}{r}0.30_{-0.08}^{+0.08} \\
0.08\end{array}$ & $2.15 \pm 0.66 \times 10^{3}$ & $9.73 \pm 0.25 \times 10^{2}$ & 1.06 & $299 / 270$ \\
\hline $10 ! 3-12 ! 9$ & $7.64_{-0.79}^{+0.80}$ & $0.19_{-0.12}^{-0.12}$ & $8.50 \pm 0.32 \times 10^{2}$ & $3.96 \pm 0.32 \times 10^{2}$ & 1.17 & $322 / 270$ \\
\hline $12 ! 9-16 ! 8$ & $5.83_{-0.86}^{+1.10}$ & 0.2 (fix) & $6.88 \pm 0.48 \times 10^{2}$ & $2.72 \pm 0.23 \times 10^{2}$ & 0.84 & $374 / 358$ \\
\hline $16^{\prime} 8-24^{\prime} 6$ & $\begin{array}{r}-0.80 \\
3.37_{-0.98}^{+1.93}\end{array}$ & 0.2 (fix) & $1.42 \pm 0.24 \times 10^{2}$ & $5.34 \pm 0.25 \times 10^{1}$ & 0.91 & $267 / 217$ \\
\hline $24 ! 6-311^{\prime} 1$ & $1.08_{-0.37}^{+0.62}$ & 0.2 (fix) & $1.53 \pm 1.15 \times 10^{1}$ & $5.45 \pm 0.15$ & 0.94 & $227 / 223$ \\
\hline $31 !^{\prime} 1-38 .{ }^{\prime} 8$ & $1.08_{-0.038}^{+0.41}$ & 0.2 (fix) & $7.24 \pm 6.30$ & $2.45 \pm 0.15$ & 0.88 & $281 / 223$ \\
\hline \multicolumn{7}{|c|}{ CONTAMI 10\% RED } \\
\hline $00^{\prime}-2.5$ & $8.27^{+0.10}$ & $0.34_{-0.02}^{+0.02}$ & $1.81 \pm 0.01 \times 10^{6}$ & $7.71 \pm 0.30 \times 10^{4}$ & 0.93 & $760 / 604$ \\
\hline $2.5-5,0$ & $9.19^{+0.19}$ & $0.28^{-0.02}$ & $3.89 \pm 0.05 \times 10^{5}$ & $1.65 \pm 0.06 \times 10^{4}$ & 0.94 & $733 / 604$ \\
\hline $5 ! 0-7 ! 8$ & $9.67_{-0.38}^{+0.38}$ & $0.24_{-0.05}^{+0.05}$ & $7.50 \pm 0.13 \times 10^{4}$ & $3.32 \pm 0.06 \times 10^{3}$ & 1.04 & $683 / 604$ \\
\hline $7 ! 8-10 ! 3$ & $8.52_{-048}^{+0.68}$ & $0.30_{-0.09}^{+0.09}$ & $2.12 \pm 0.67 \times 10^{3}$ & $9.62 \pm 0.32 \times 10^{2}$ & 1.07 & $299 / 270$ \\
\hline $10 ! 3-12 ! 9$ & $7.92_{-0.79}^{+0.84}$ & $0.19_{-0.13}^{+0.13}$ & $8.29 \pm 0.32 \times 10^{2}$ & $3.92 \pm 0.33 \times 10^{2}$ & 1.19 & $317 / 270$ \\
\hline $12 ! 9-16 ! 8$ & $6.06_{-0.92}^{+1.23}$ & 0.2 (fix) & $6.80 \pm 0.40 \times 10^{2}$ & $2.67 \pm 0.23 \times 10^{2}$ & 0.84 & $372 / 358$ \\
\hline $16^{\prime} 8-24^{\prime} 6$ & $3.27_{-0.93}^{+1.71}$ & 0.2 (fix) & $1.45 \pm 0.44 \times 10^{2}$ & $5.45 \pm 0.28 \times 10^{1}$ & 0.90 & $263 / 217$ \\
\hline $24 .^{\prime} 6-311^{\prime} 1$ & $0.97_{-0.25}^{+0.35}$ & 0.2 (fix) & $2.23 \pm 1.15 \times 10^{1}$ & $8.25 \pm 0.25$ & 0.97 & $228 / 223$ \\
\hline $31 !^{\prime} 1-38 .{ }^{\prime} 8$ & $1.04_{-0.32}^{+0.26}$ & 0.2 (fix) & $1.10 \pm 0.58 \times 10^{1}$ & $3.85 \pm 0.25$ & 0.87 & $275 / 223$ \\
\hline
\end{tabular}

\section{Discussion}

Suzaku performed four pointing observations in A2142 and its outside regions along the merger axis. The temperature was measured out to the virial radius $\left(r_{200} \sim 2.5 \mathrm{Mpc}\right)$ for the first time. The ICM temperature was found to drop from about 9 $\mathrm{keV}$ around the center to about $3 \mathrm{keV}$ at $r_{200}$. We detected no significant signal from the WHIM and set upper limits of its over-density. We will attempt to evaluate the cluster properties (temperature, electron density, and entropy) and discuss their implications.

\subsection{Temperature Profile}

Some numerical simulations (Ettori et al. 2004; Borgani et al. 2004) predicted that the intracluster gas temperature drops to about $50 \%$ of the central temperature around $r_{200}$ under hydrostatic equilibrium. Those results reproduced observed 
(a) Temperature

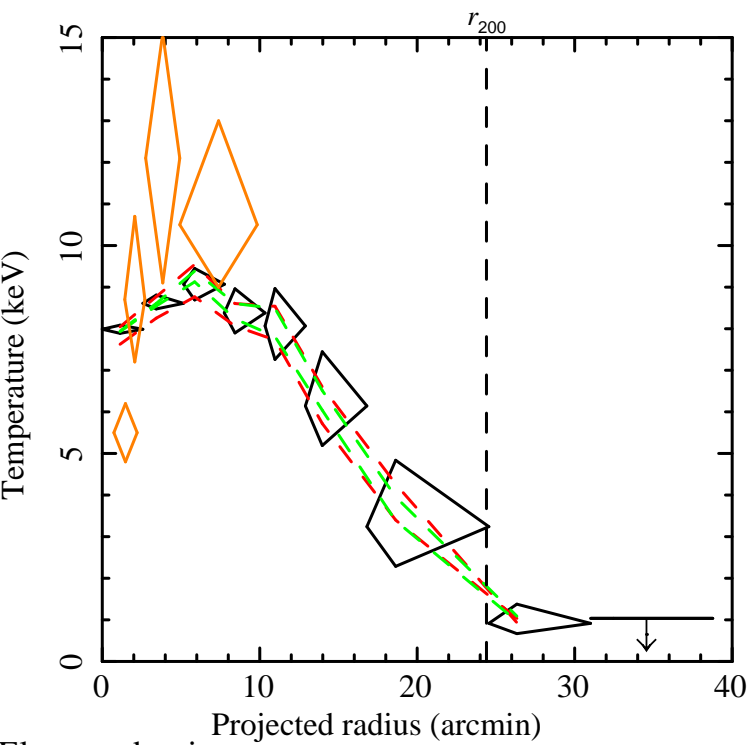

(b) Electron density

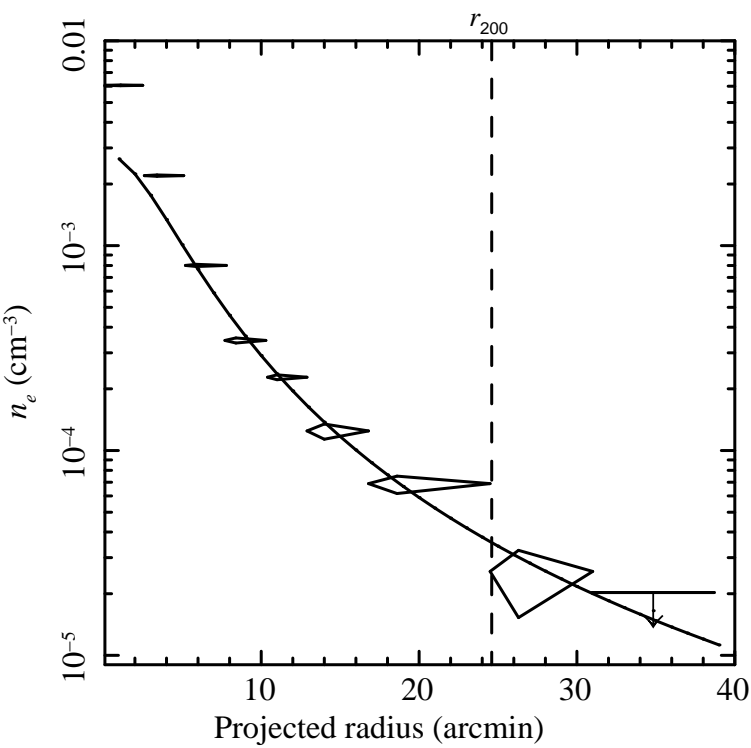

Fig. 4. Radial profiles for (a) temperature, (b) 3-dimensional electron density. In the temperature plot, the uncertainty range due to the combination of $\pm 3 \%$ variation of the NXB level and the maximum/minimum fluctuation of CXB is shown by two green dashed lines. In a similar way, the uncertainty range due to the effect of contamination on the blocking filter is shown by red lines. Orange diamonds show Chandra data from Markevitch et al. (2000).

temperature profiles to about $0.5 r_{200}$ (De Grandi \& Molendi 2002; Vikhlinin et al. 2005; Pratt et al. 2007). In particular, recent XMM-Newton (Pratt et al. 2007) results showed temperature profiles out to $0.8 r_{200}$.

We compare here the observed temperature profile of A2142 with the pro?le of other clusters that have been observed with Suzaku. Table 8 shows the list of clusters for which Suzaku measured the temperature out to $r_{200}$. Their temperature profiles are shown in figure 6. All the temperatures are normalized by the flux-weighted average of each clusters. The results clearly show a systematic drop of the temperature by a factor
Table 7. WHIM upper limit

\begin{tabular}{|c|c|c|c|c|c|c|c|c|}
\hline Region & $I_{\mathrm{OVII}}^{*}$ & $I_{\mathrm{OVm}}^{*}$ & $\delta_{\text {OVII }}$ & $\delta_{\mathrm{OVIII}}$ & $I_{\mathrm{OVI}}^{*}$ & $I_{\mathrm{OVm}}^{*}$ & $\delta_{\mathrm{OVII}}$ & $\delta_{\mathrm{OVIII}}$ \\
\hline & \multicolumn{4}{|c|}{ BGD OFF3 } & \multicolumn{4}{|c|}{ BGD TCRB } \\
\hline Takei et al. & $<1.1$ & $<3.0$ & $<270$ & & & & & \\
\hline $0^{\prime}-2 \cdot 5$ & 83.60 & 16.50 & 3311 & 1470 & 85.30 & 17.24 & 3344 & 1503 \\
\hline $2 ! 5-5.0$ & 20.28 & 3.79 & 1630 & 705 & 21.85 & 4.51 & 1693 & 769 \\
\hline 5.0-7!.8 & 3.58 & 1.01 & 685 & 364 & 5.14 & 1.38 & 821 & 425 \\
\hline $7 ! 8-10 ! 3$ & 0.92 & 0.68 & 347 & 299 & 1.48 & 1.06 & 440 & 373 \\
\hline $10 ! 3-12 ! 9$ & 0.79 & 0.30 & 321 & 199 & 1.41 & 0.44 & 429 & 240 \\
\hline $12 ! 9-16: 8$ & 1.34 & 1.82 & 419 & 489 & 2.56 & 2.94 & 579 & 621 \\
\hline $16 ! 8-24 ! 6$ & 0.73 & 0.64 & 309 & 289 & 1.66 & 1.32 & 466 & 416 \\
\hline $24 ! 6-31 ! 1$ & 0.60 & 0.62 & 281 & 284 & 1.66 & 1.46 & 466 & 437 \\
\hline $31{ }^{\prime} 1-38: 8$ & 0.57 & 1.01 & 275 & 363 & 1.08 & 1.32 & 377 & 415 \\
\hline
\end{tabular}

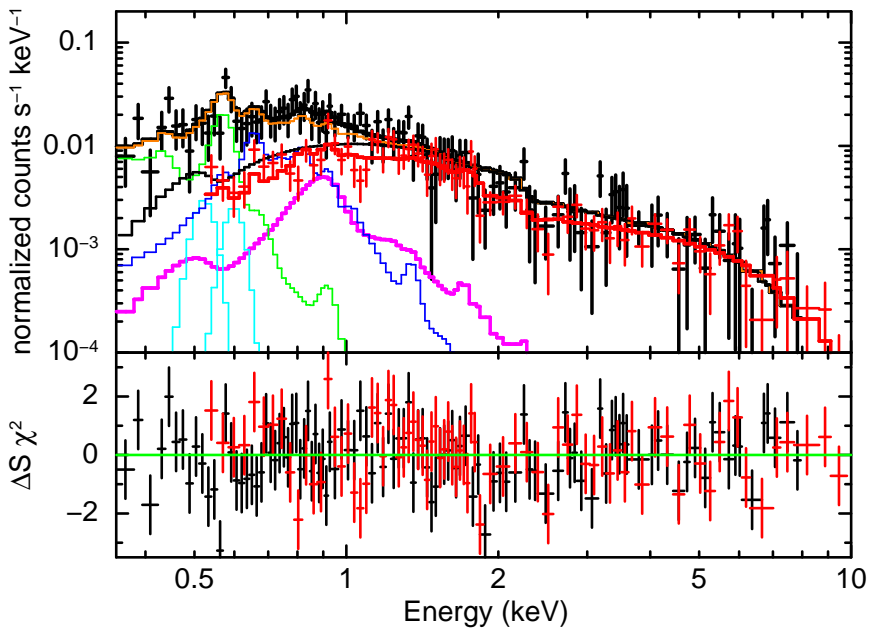

Fig. 5. Fits for the constraint on the intensities of the $I_{\mathrm{OVII}}$ and $I_{\mathrm{OVIII}}$ lines in the $31^{\prime} 1-38^{\prime} .8$ region. The $I_{\mathrm{OVII}}$ and $I_{\mathrm{OVIII}}$ emission lines are shown as a cyan lines, and the notations of the other lines are same as shown in figure 3 .

of 3-5 from the center to $r_{200}$.

Among these clusters, A1689 shows a significant directional difference in the sense that the temperature drop is small along the filament direction (Kawaharada et al. 2010). Another system A1413 also indicates a somewhat flatter temperature profile, and the measured direction is along the longer axis of its X-ray elongation (Hoshino et al. 2010).

Burns et al. (2010) discuss a non-equilibrium effect on the temperature profile based on N-body + hydrodynamic simulations. Those simulations indicate cluster temperature decline by factor of $\sim 3$ at $r_{200}$, consistent with the feature observed for two nearby clusters, PKS 0745-191 and A 1795 (George et al. 2008; Bautz et al. 2009). Burns et al. (2010) approximated the average temperature profile by a function,

$$
\frac{T}{T_{\mathrm{avg}}}=A\left[1+B\left(\frac{r}{r_{200}}\right)\right]^{\beta} \text {. }
$$

They obtained the best-fit values as $A=1.74 \pm 0.03, B=$ $0.64 \pm 0.10, \beta=-3.2 \pm 0.4$ for the two cluster data. In figure 6 , we plotted model prediction temperature profile. Black dotted curve shows the best-fit temperature profile, and dashed lines show $1 \sigma$ error range reported by Burns et al. (2010). The 
relation of Burns et al. (2010) represents the temperature profiles for the 6 clusters fairly well. This approximate "universal" temperature profile suggests that clusters generally hold selfsimilar relation even near $r_{200}$, where some temporary effects caused by infalling matter may be seen. Note that the temperature drop in the filament direction of A1689 (not shown in Fig 6) is flatter than the average A1689 profile, suggesting a very efficient heating going on in the filament direction as compared with typical clusters.

Table 8. Cluster samples and those properties

\begin{tabular}{ccccc}
\hline \hline Cluster & $z$ & Ref. & $\begin{array}{c}k\langle T\rangle \\
{[\mathrm{keV}]}\end{array}$ & $\begin{array}{c}r_{200} \\
{[\mathrm{Mpc}](\operatorname{arcmin})}\end{array}$ \\
\hline Abell 2142 & 0.090 & This work & 8.6 & $2.46(24.6)$ \\
PKS 0745-191 & 0.103 & George et al. 2008 & 7.0 & $2.21(19.6)$ \\
Abell 2052 & 0.036 & Tamura et al. 2008 & 3.2 & $1.54(36.7)$ \\
Abell 2204 & 0.152 & Reiprich et al. 2009 & 8.7 & $2.40(13.2)$ \\
Abell 1795 & 0.063 & Bautz et al. 2009 & 5.3 & $1.96(26.9)$ \\
Abell 1413 & 0.143 & Hoshino et al. 2010 & 7.4 & $2.24(14.8)$ \\
Abell 1689 & 0.183 & Kawaharada et al. 2010 & 9.3 & $2.44(13.3)$ \\
Perseus & 0.018 & Simionescu et al. 2011 & 6.5 & $2.22(103.1)$ \\
\hline
\end{tabular}

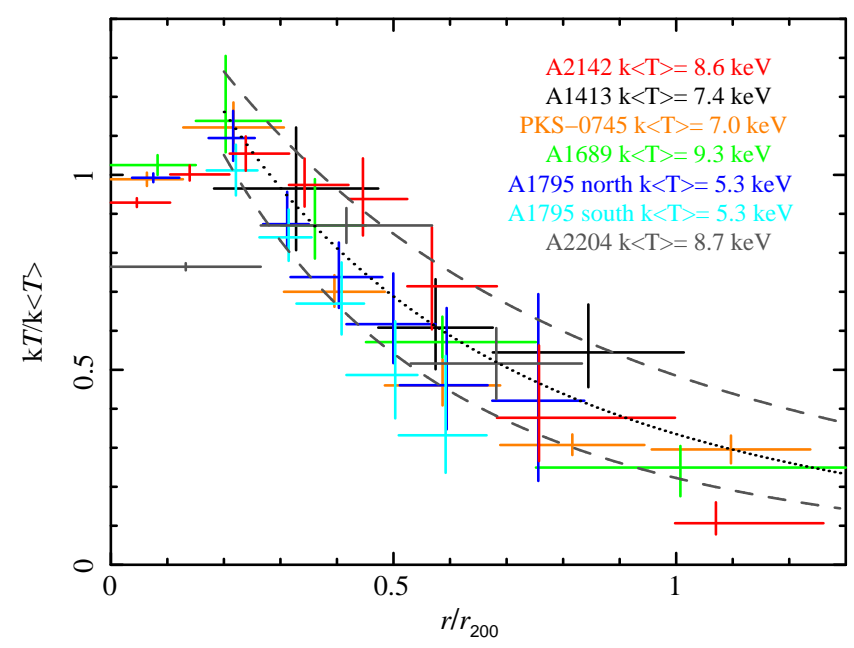

Fig. 6. Scaled projected temperature profiles. The profiles have been normalized to the mean temperature. The $r_{200}$ derived from Henry et al. (2009). Dotted line show simulation result Burns et al. (2010). Two gray dashed lines show standard deviation.

All the clusters in figure 6 are morphologically relaxed systems, even though all of them show an elliptical shape to some extent. Therefore, we may regard the model temperature profile to be a typical feature for clusters having relaxed morphologies. We note that the present A2142 profile shows a smooth temperature decline with radius, even though it is a merger cluster characterized by clear cold fronts within $3^{\prime}\left(0.11 r_{200}\right)$ from the center.

We note that when we fit the temperature profiles with the above formula 2, A2142 data are characterized by $\beta=$ $-5.8 \pm 0.8$, which indicates a steepr slpe than the average, $\beta=-3.2 \pm 0.4$. On the other hand in A1689, the filament direction shows a flatter temperature profile (Kawaharada et al. 2010) than the average curve. It has been discussed that A1689 still holds the heating feature caused by recent matter infall. The same process may be working in A1413 which also shows somewhat flatter temperature distribution (Hoshino et al. 2010) even though the presence of filament is not clear in the observed direction.

Even though flatter temperature profiles are regarded as a sign of recent matter infall, steeper temperature curves such as seen in A 2142 and abrupt temperature drops, as seen in A 3667 and Virgo Cluster can also be a sign of recent heating (Finoguenov et al. 2010; Urban et al. 2011). This point will be discussed further in the next section. Therefore, simple steepness of the temperature profile seems to carry somewhat degenerate information about the cluster evolution.

\subsection{Entropy Profiles}

The entropy of ICM is used as an indicator of the energy acquired by the gas. We will refer to "entropy" of the ICM by $K=k T n_{e}^{-2 / 3}$ following the recent convention. Numerical simulations indicate that a self-similar growth of clusters commonly show entropy profiles approximated by $r^{1.1}$ up to $r_{200}$, excluding the cool core region (Voit et al. 2003). Recent XMMNewton results on the entropy profiles of 31 clusters showed a median slope of 0.98 out to about $r_{500}$ which is approximately $0.5 r_{200}$ (Pratt et al. 2010). The slope also showed positive correlation with the average temperature. They also found that morphologically disturbed clusters show a large scatter $(0.5-2.0)$ in the slope. Suzaku has extended the entropy measurement close to $r_{200}$ for several clusters, and showed a flattening or even a decrease at $r \gtrsim 0.5 r_{200}$ as mentioned earlier. Discussion has been made that the ICM may not be under equilibrium in the cluster outer regions (Hoshino et al. 2010).

Figure 7 shows the entropy profile of A2142 based on the present Suzaku measurements. The solid line indicates the slope of 1.1. The entropy slope is consistent with this value in the radius range $0.1-0.4 r_{200}\left(2.5-10^{\prime}\right)$. The slope becomes flatter at $r>0.4 r_{200}$ and goes negative near $r_{200}$. This feature is more clearly seen in figure $7(\mathrm{~b})$, which shows the ratio to the $r^{1.1}$ curve which can be fitted for the inner profile, $r<0.4 r_{200}$. Including the previous results for PKS0745-191 and A1413, flattening of the entropy profile in such outer regions looks to be a common feature.

As for the cause of the entropy drop relative to the predicted $r^{1.1}$ relation, two possibilities have been pointed out so far. One is the non-equilibrium effect (Hoshino et al. 2010; Burns et al. 2010) and the other is the clumpiness (Simionescu et al. 2011), both working only in the outer regions. These two conditions are not mutually exclusive but may be closely related with each other.

Regarding the effect of clumpiness, Nagai (2011) examined with numerical simulations how much clumping in accreting gas could cause overestimation of gas density since the emissivity scales as density squared. This density overestimation gives lower entropy values, hence resulting in a flattening around $r_{200}$. Results of Nagai (2011) show that the entropy drops by $10-15 \%$ from the true value at around $r_{200}$. In the A2142 case, the observed entropy curve starts to flatten around $r_{500}$ and decreases to less than $40 \%$ of the $r^{1.1}$ extention at $r_{200}\left(\sim 25^{\prime}\right)$. This large suppression of the entropy seems too large according to the simulation. Therefore, clumping is not likely to be the only or the dominant source of the entropy suppression. 
(a) Entropy

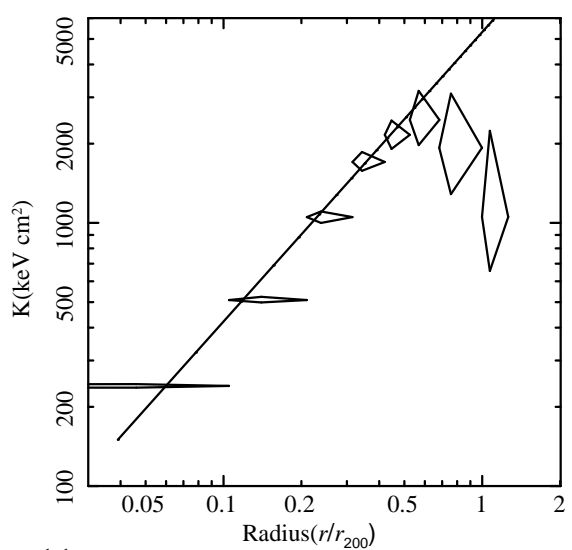

(b) Ratio to $r^{1.1}$ consistent with $T_{e} / T_{\text {gas }}$

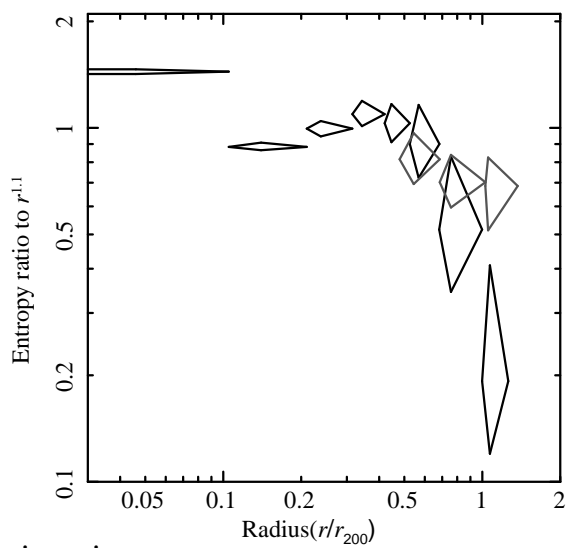

(c) Equilibrium time

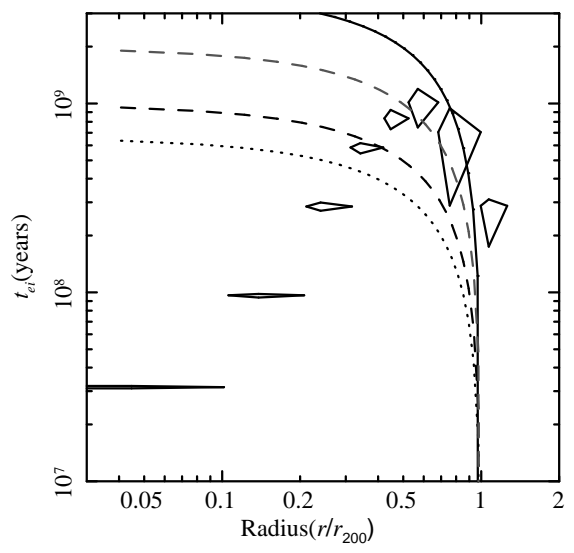

Fig. 7. (a) Entropy profile: Diamonds show Suzaku results, and solid straight line shows the universal trend of the entropy curve $\sim r^{1.1}$, reported by Voit (2005). (b) Entropy ratio relative to the $r^{1.1}$ profile. Gray diamonds show the result of A1413 (Hoshino et al. 2010) (c) $t_{e i}$ (diamonds) as a function of radius, compared with estimated $t_{\text {elapsed }}$ (solid line). Different lines show different shock speeds (gray dash:400 km $\mathrm{s}^{-1}$, dash: $800 \mathrm{~km} \mathrm{~s}^{-1}$, dot: $1200 \mathrm{~km} \mathrm{~s}^{-1}$ ).

\subsection{Ion-Electron Relaxation}

The entropy profile of A2142 shows a flatter slope than the predicted one based on numerical simulations, and even a negative slope is seen in the outermost region. Kawaharada et al. (2010) showed a flat entropy profile in the filament direction of A1689 and discussed effects from surrounding environments.
If we make a simple assumption that the entropy profile of ICM should follow $r^{1.1}$ (Voit et al. 2003) under an equilibrium, the present result for A2142 suggests that electrons in the cluster outer region is not in the equilibrium.

Hoshino et al. (2010) discussed the possible difference between ion and electron temperatures in the outer low-density region, where the relaxation time scale is inversely proportional to particle density. We follow their estimation and look into the behavior of ion $\left(T_{i}\right)$ and electron $\left(T_{e}\right)$ temperatures. The following assumptions are made.

1. Ions are initially heated through accretion shocks at $r_{200}$.

2. Ions achieve thermal equilibrium with a timescale of ionion relaxation, $t_{i i}$, after this heating.

3. Thermal energy is transferred from ions to electrons through Coulomb collisions. This process takes a timescale of $t_{e i}$ which is much longer than both $t_{i i}$ and the electron-electron relaxation time $t_{e e}$.

The relation between $T_{i}$ and $T_{e}$ can be estimated in the following way after Hoshino et al. (2010). We look into a position-dependent time, $t_{\text {elapsed }}$, which is a rough measure of the time elapsed from the shock heating. We assume that the shock heating takes place at the virial radius and that the shock wave propagates with a constant speed (400, 800, $1200 \mathrm{~km}$ $\mathrm{s}^{-1}$ ) in ICM toward inner regions. This $t_{\text {elapsed }}$ can be compared with the equilibration timescale $t_{e i}$, and we may assume that electrons are fully heated up when $t_{\text {elapsed }}>t_{e i}$. In figure 7(c), we compare $t_{\text {elapsed }}$ with $t_{e i}$ as a function of radius. Each curves show the position-dependent $t_{\text {elapsed }}$, assuming that the gas falls through either free-fall or constant velocities. The equilibration timescale $t_{e i}$ is significantly longer than $t_{\text {elapsed }}$ in the cluster outskirts at $r>20^{\prime}$, suggesting that the ion temperature can be higher than the electron temperature. This feature is essentially the same in A 1413 as reported by Hoshino et al. (2010).

Theoretical studies of non-equilibrium ionization state and an electron-ion two-temperature structure of ICM in merging galaxy clusters have been carried out (Rudd \& Nagai 2009; Akahori \& Yoshikawa 2010). They show that in merging clusters, $T_{e}$ is lower than the average temperature by $20-30 \%$. Following Hoshino et al. (2010), we estimate the possible deviation of $T_{e}$ by assuming that the average temperature is given by the entropy and density as $k T_{\text {gas }}=K n^{2 / 3}$, and further that the entropy $K$ follows the power-law profile $\propto r^{1.1}$ to $r_{200}$ (Voit et al. 2003). Figure 7(b) shows thus estimated ratio $T_{e} / T_{\mathrm{gas}}$. Previous A1413 results by Hoshino et al. (2010) are shown for compariton. A2142 values are lower than A1413 at $r_{200}$, which may reflect stronger suppresion of $T_{e}$ in merging clusters. The $T_{e} / T_{\text {gas }}$ value in A2142 is $0.51_{-0.17}^{+0.31}$ at $r_{200}$, which is in good agreement with the theoretical result for unrelaxed clusters (Rudd \& Nagai 2009).

Even though this feature suggests that $T_{e}$ is substantially lower than the average gas temperature around $r_{200}$, we have to note that the temperature profile of A2142 agrees with those for other relaxed clusters as seen in figure 6. This may suggest that the entropy drop is rather due to high $n_{e}$ around $r_{200}$. However, as shown in figure 4 (b), the observed $n_{e}$ profile also follows a smooth $\beta$-model and no peculiar hump is seen in the outer region. It may be that the outer region of A2142 has not experienced strong merger recently, but then the entropy 
"saturation" in the outer region will have to have a more universal origin. We certainly need to look into this problem with a wider range of cluster sample regarding the cluster size and morphology.

\subsection{Electron Density Profile}

The electron number density profile in figure 4 shows a decrease down to $\sim 10^{-5} \mathrm{~cm}^{-3}$ around the virial radius. ROSAT study showed that the electron density profile could be fitted well except for the cluster center with the $\beta$-model, after modification for the cluster ellipticity (Henry \& Briel 1996). In the outskirts of relaxed clusters, many systems indicate a density profile of $n_{e} \propto r^{-2.2}$ (Zhang et al. 2006). Also, $n_{e} \propto r^{-1.8 \pm 0.28}$ is obtained for the REXCESS sample (Croston et al. 2008)

The measured A2142 density profile is fitted with a powerlaw model with an index of $-1.98 \pm 0.13$ for the entire radial range and $-2.53 \pm 0.25$ in the outskirts only $\left(r>0.5 r_{200}\right)$. These results agree with the previous studies for other clusters, and with the predicted profile of $n_{e} \propto r^{-2.5}$ based on the $\beta$-model $(\beta=0.85)$ in the outer region.

Recently, Eckert et al. (2011) pointed out a factor of about 3 discrepancy in the surface brightness in the outer region of PKS 0745-191 between Suzaku and ROSAT results. They argue that it is most likely to be caused by an incorrect subtraction of the Galactic emission for the Suzaku data. As for A2142, ROSAT PSPC observed its emission to $r_{200}$ (Henry \& Briel 1996) and showed $n_{e} \sim 5 \times 10^{-5} \mathrm{~cm}^{-3}$ at $r_{200}$. The present $n_{e}$ profile, shown in figure 4 , indicates a lower but consistent value with the ROSAT one within a factor of $\sim 1.5$. Therefore, our subtraction of the Galactic background in the nearby sky region is considered to give reliable results.

\subsection{Mass Estimation to $r_{200}$}

We estimate the gravitational mass of A2142 to $r_{200}$ based on the observed temperature and density profiles. Here, we assume hydrostatic equilibrium and spherical symmetry, and calculate the gravitational mass within 3-demensional radius $R$ with the following formula (Fabricant et al. 1980),

$$
M_{R}=-\frac{R^{2}}{\rho_{g} G} \frac{d P_{g}}{d R}=-\frac{k T R}{\mu m_{p} G}\left(\frac{d \ln \rho_{g}}{d \ln R}+\frac{d \ln T}{d \ln R}\right),
$$

where $G$ is the gravitational constant, and $\mu(\approx 0.6)$ is the mean molecular weight of gas.

In figure 8, solid diamonds show the gravitational mass of A2142 based on the observed temperature and density profiles. Two solid vertical lines (black and gray) indicate the mass derived from the previous weak lensing studies which are summarized in table 9 (Okabe \& Umetsu 2008; Umetsu et al. 2009). Dashed-line diamonds show gravitational mass using the $\beta$-model density profile together with the observed temperature gradient. The resultant mass agrees well with the previous studies around the virial radius.

We compare the parameters of an NFW profiles, defined as

$$
\rho=\frac{\rho_{s}}{\left(r / r_{s}\right)\left(1+r / r_{s}\right)^{2}}
$$

where $\rho_{s}$ is central density parameter and $r_{s}$ is the scaled radius. Figure 8(b) show the resultant values of differential mass density $\left(c=2.8_{-1.2}^{+1.0}\right)$, which reflect the dark matter potential.
Our differential mass density profile is consistent with the previous weak-lensing result which shows $c=4.26_{-0.63}^{+0.71}$ (Umetsu et al. 2009).

We can evaluate the contribution of non-thermal pressure by comparing the X-ray and the weak-lensing masses. The Xray to lensing mass ratio is $0.90_{-0.35}^{+0.48}$, which indicates that, by taking the lowest boundary value, the maximum non-thermal pressure can be $45 \%$. As shown in $\$ 6.3$, we discussed the possible difference between ion and electron temperatures as the cause of the entropy drop from the $r^{1.1}$ relation. In order for the entropy profile to follow the $r^{1.1}$ relation after electron temperature reaches the ion temperature, ion temperature should have higher temperature than electrons. The ratio of electron to ion temperature is estimated to be $0.51_{-0.17}^{+0.31}$ around the virial radius (see fig7b: this value also indicates the ratio of electron to ion temperatures). Then, since the gravitational force is balanced with the sum of electron and ion pressure in hydrostatic equilibrium, the gravitational mass would rise roughly by the same factor. Although this leads to poorer match between X-ray and lensing mass, they are still barely consistent within statistical and systematic uncertainties.

We note that non-thermal pressure can make additional contribution to the cluster mass estimation. Dynamical effects such as turbulence and bulk motion of ICM are not yet measured, but can give significant effects in the cluster outer regions. Some numerical simulations (Nagai et al. 2007; Piffaretti \& Valdarnini 2008) predicted that such non-thermal pressure could add up $15-30 \%$ of the cluster mass.

We also looked into the gas mass distribution using the obtained electron density profile and showed the results in figure 8(a) with black crosses. Here, the gas mass does not include the stellar mass. The resultant gas mass fraction at $r_{200}$ is $14.4_{-4.1}^{+7.1 \%}$. This agrees with the expected hot-gas fraction $(15 \%)$ in the universe (Komatsu et al. 2011), and consistent with the previous result, $\sim 18 \%$ at $22^{\prime} .8$, incorporating the weak lensing and Sunyaev and Zel'dovich observations (Umetsu et al. 2009). This indicates that, in A2142, there is no strong need to invoke the gas clumpiness to account for the gas mass fraction as in the Perseus cluster (Simionescu et al. 2011).

Table 9. Mass estimation of A2142

\begin{tabular}{ccc}
\hline Reference & $r$ & $M_{200}$ \\
& $\operatorname{arcmin}$ & $10^{14} M_{\odot}$ \\
\hline Okabe \& Umetsu 2008 & 22.2 & $13.7 \pm 6.0$ \\
Umetsu et al. 2009 & 22.2 & $12.3_{-2.0}^{+3.0}$ \\
This work (Observed gas density profile) & $22.5 \pm 3.9$ & $11.1_{-3.1}^{+5.5}$ \\
This work $(\beta$-model gas density profile) & $22.5 \pm 3.9$ & $10.4_{-2.9}^{+5.2}$ \\
\hline
\end{tabular}

\section{Summary}

We observed Abell 2142 in the direction of the possible merger axis with Suzaku and detected the ICM emission up to the virial radius $r_{200}(2.5 \mathrm{Mpc} \sim 24$. 6$)$. We derived radial profiles of temperature, electron density, and entropy, and compared these properties with the previous results for other relaxed clusters. We summarize the main features of A2142 as follows; 
(a) Total integrated gravitational mass

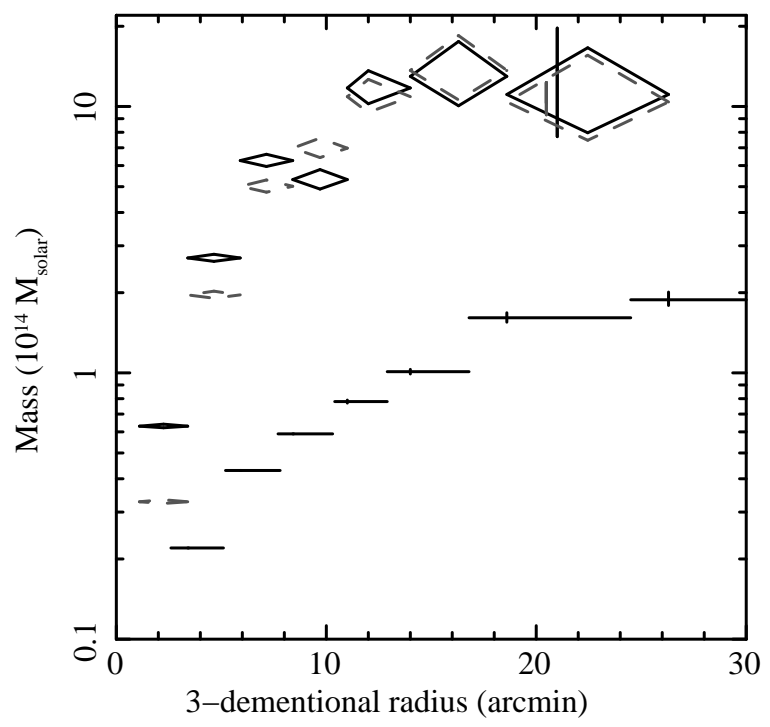

(b) Differential mass density

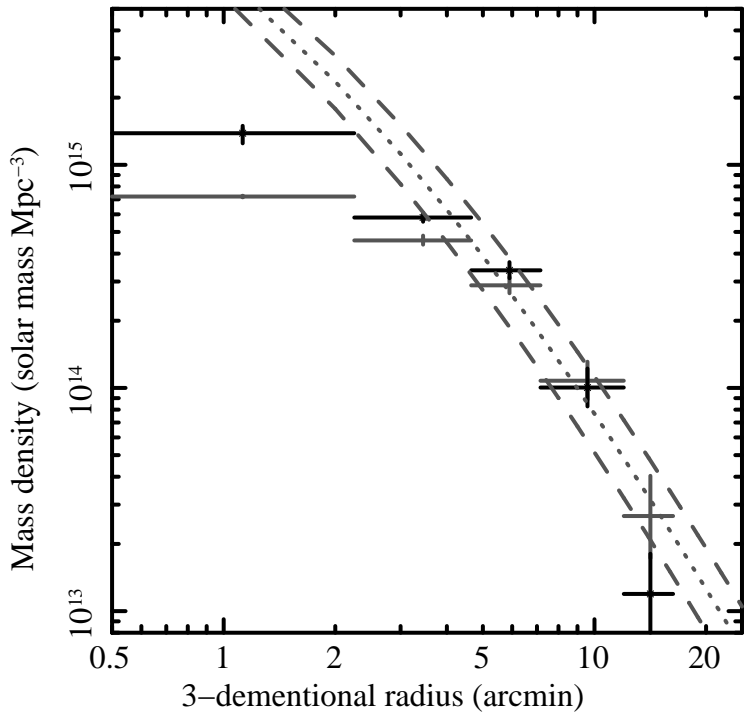

Fig. 8. (a) Total integrated gravitational mass $M_{<R}$ profile of A2142. Black diamonds show gravitational mass profile estimated from the Suzaku data. Gray dashed diamonds show a profile assuming the electron density to follow $\beta$ model distribution with $\beta=0.85$. Black and gray vertical lines show masses estimated by weak-lensing analysis summarized in table 9 (Okabe \& Umetsu 2008; Umetsu et al. 2009). Black cross show gas mass profile estimated from the observed electron density profiles. (b) Same as (a), but for differential mass density. We ignore the data around $10^{\prime}$, because it gives an unphysical negative value, Dotted curve show NFW-model results by Umetsu et al. (2009). Two dashed lines show standard deviation.

- The ICM temperature gradually decreases toward the outer region from about $10 \mathrm{keV}$ at $0.2 r_{200}$ to about $4 \mathrm{keV}$ at $r_{200}$.

- The temperature profile in the outer region of A2142 agrees well with the results of other clusters observed by Suzaku.

- The average temperature profile for different clusters can be described by the formula by Burns et al. (2010) up to $r_{200}$, suggesting that non-thermal pressure support is significant in the outer regions.

- The electron density profile decreases down to $10^{-5} \mathrm{~cm}^{-3}$ at the virial radius and well agrees with the $\beta$ model with $\beta=0.85$.

- The entropy profile within about $0.4 r_{200}$ follows $r^{1.1}$, predicted by the accretion shock heating model. The profile becomes flatter and finally shows a negative slope around $r_{200}$, suggesting significant deviation from the equilibrium condition.

- Based on the temperature and entropy profiles, and required relaxation time, we discuss that $T_{i}$ is likely higher than $T_{e}$ in the outer region of A2142.

- The derived mass profile is in agreement with the weaklensing mass. The difference between X-ray and lensing masses allowed by the error can explain the contribution of the non-equilibrium effect.

Resent Suzaku results on the temperature and entropy measurements to $r_{200}$, including the A2142 ones, jointly suggest that ICM in cluster outskirts is deviating from thermal equilibrium in the sense that the electron temperature could be significantly lower than the ion temperature. This suggests that the ICM around $r_{200}$ has experienced bulk motions and/or turbulence within a time scale of about $10^{9} \mathrm{yr}$. Such gas motions with a velocity of a few hundred to thousand $\mathrm{km} \mathrm{s}^{-1}$ can be observable in future by X-ray microcalorimeters, such as by SXS instrument on ASTRO-H (Mitsuda et al. 2010) which has 20 30 times higher energy resolution than CCD instruments. Since the FOV of SXS is rather small $\left(3^{\prime} \times 3^{\prime}\right)$, expected counts from $\mathrm{Fe}-\mathrm{K}$ lines are typically around 10 and 60 at $r_{200}$ and $0.5 r_{200}$, respectively, for a $300 \mathrm{ksec}$ exposure. Therefore, long exposures for selected good targets with ASTRO-H will be needed to show the dynamical features appearing in the cluster outer regions for the first time.

We are grateful to N. Okabe and K. Umetsu for information about thier Suzaku data and N. Okabe providing SDSS galaxy distribution data. H. A. was supported by Grant-in-Aid for JSPS Fellows $(22 \cdot 1582)$ and the MEXT program " Support Program for Improving Graduate School Education”. NO acknowledges financial support from Grant-in-Aid for Scientific Research No.22740124.

\section{Appendix 1. Point Source Analysis}

As for the point-source subtraction, we first analyzed the XMM-Newton data (Observation ID=0111870101, 0111870401) in which faint sources were resolved better than the Suzaku data. The data covered up to the virial radius $(\sim 2.5$ $\mathrm{Mpc}$ ). We used wavdetect tool in CIAO (CIAO version:4.0.1) to detect point sources and used surround annular region for background subtraction. We summed MOS1 and MOS2 spectra to increase statistics, and fitted by pegpowerlaw model which offered photon index and flux in selected energy band. In the outer region $(r>25$ ! 6$)$, we removed the sources from the Suzaku data which were selected by eye. In the XMMNewton case, the source extraction radius is $30^{\prime \prime}$, and the sur- 
Table 10. Informations of point source in two XMM and Suzaku observations of A2142 center, OFFSET1, OFFSET2 OFFSET3.

\begin{tabular}{cccccccc}
\hline & & \multicolumn{2}{c}{ XMM-Newton(MOS1+MOS2) } & \multicolumn{3}{c}{ Suzaku } \\
No. $^{*}$ & $(\alpha, \delta)^{\dagger}$ & Photon Index & Flux $^{\dagger}$ & $\chi^{2} /$ d.o.f & Photon Index & Flux & $\chi^{2} /$ d.o.f \\
\hline 1 & $(239.401,27.485)$ & $1.44_{-0.21}^{+0.22}$ & $1.28_{-0.34}^{+0.72}$ & $108.9 / 63$ & $1.96_{-0.23}^{+0.29}$ & $1.02_{-0.30}^{+0.37}$ & $53.0 / 52$ \\
2 & $(239.393,27.287)$ & $1.42_{-0.68}^{+0.63}$ & $0.31_{-0.31}^{+0.56}$ & $35.6 / 23$ & $1.69_{-0.14}^{+0.14}$ & $1.40_{-0.48}^{+0.52}$ & $59.0 / 53$ \\
3 & $(239.334,27.275)$ & $2.21_{-0.50}^{+0.64}$ & $1.27_{-0.18}^{+0.46}$ & $34.0 / 31$ & $2.08_{-0.13}^{+0.15}$ & $1.88_{-0.42}^{+0.46}$ & $75.0 / 53$ \\
4 & $(239.283,27.366)$ & - & - & $-/-$ & $1.64_{-0.18}^{+0.23}$ & $2.32_{-0.59}^{+0.59}$ & $74.0 / 52$ \\
5 & $(239.532,27.351)$ & - & - & $-/-$ & $0.94_{-0.74}^{+0.54}$ & $2.28_{-1.79}^{+6.51}$ & $58.0 / 51$ \\
6 & $(239.295,27.605)$ & - & - & $-/-$ & $1.89_{-0.18}^{+0.19}$ & $0.89_{-0.18}^{+0.19}$ & $70.0 / 54$ \\
8 & $(238.922,27.686)$ & - & - & $-/-$ & $1.93_{-0.10}^{+0.10}$ & $3.23_{-0.39}^{+0.41}$ & $60.0 / 54$ \\
9 & $(239.012,27.773)$ & - & - & $-/-$ & $1.63_{-0.14}^{+0.15}$ & $1.65_{-0.26}^{+0.28}$ & $52.0 / 54$ \\
\hline
\end{tabular}

$*$ :Serial number for point source.

$\ddagger$ The $2.0-10.0 \mathrm{keV}$ flux in units of $10^{-13} \mathrm{erg} \mathrm{cm}^{-2} \mathrm{~s}^{-1}$

rounding background ring in estimating the flux is defined by $30^{\prime \prime}-60^{\prime \prime}$, respectively. In the Suzaku case, those are $1^{\prime}$ and $1^{\prime}-2^{\prime}$, respectively. We found 3 sources in the XMM-Newton data whose fluxes were higher than $3 \times 10^{-14} \mathrm{erg} \mathrm{cm}^{-2} \mathrm{~s}^{-1}$ in the energy range $2-10 \mathrm{keV}$. In the Suzaku case, we found 5 sources whose fluxes were higher than $8 \times 10^{-14} \mathrm{erg} \mathrm{cm}^{-2} \mathrm{~s}^{-1}$ in the energy range $2-10 \mathrm{keV}$.

\section{References}

Akahori, T., \& Yoshikawa, K. 2010, PASJ, 62, 335

Anders, E., \& Grevesse, N. 1989, Geochim. Cosmochim. Acta, 53, 197

Bautz, M. W., et al. 2009, PASJ, 61, 1117

Borgani, S., et al. 2004, MNRAS, 348, 1078

Burns, J. O., Skillman, S. W., \& O'Shea, B. W. 2010, ApJ, 721, 1105

Cavaliere, A., Lapi, A., \& Fusco-Femiano, R. 2011, A\&A, 525, A110

Cen, R., \& Ostriker, J. P. 2006, ApJ, 650, 560

Croston, J. H., et al. 2008, A\&A, 487, 431

De Grandi, S., \& Molendi, S. 2002, ApJ, 567, 163

Dickey, J. M., \& Lockman, F. J. 1990, ARA\&A, 28, 215

Eisenstein, D. J., et al. 2005, ApJ, 633, 560

Eckert, D., Molendi, S., Gastaldello, F., \& Rossetti, M. 2011, A\&A, 529, A133

Ettori, S., De Grandi, S., \& Molendi, S. 2002, A\&A, 391, 841

Ettori, S., et al. 2004, MNRAS, 354, 111

Evrard, A. E., Metzler, C. A., \& Navarro, J. F., 1996, ApJ, 469, 494

Fabricant, D., Lecar, M., \& Gorenstein, P. 1980, ApJ, 241, 552

Forman, W., \& Jones, C. 1982, ARA\&A, 20, 547

Finoguenov, A., Sarazin, C. L., Nakazawa, K., Wik, D. R., \& Clarke, T. E. 2010 , apj, 715, 1143

Fujita, Y., Tawa, N., Hayashida, K., Takizawa, M., Matsumoto, H., Okabe, N., \& Reiprich, T. H. 2008, PASJ, 60, 343

George,M.R.,Fabian, A. C., Sanders,J. S., Young., A. J., and Russell, H. R., 2008, MNRAS, 395, 657

Hayashida, K., Inoue, H., Koyama, K., 1989, PASJ, 41, 1373

Henry, J. P., \& Briel, U. G. 1996, ApJ, 472, 137

Henry, J. P. 2000, ApJ, 534, 565

Henry, J. P., Evrard, A. E., Hoekstra, H., Babul, A., \& Mahdavi, A. 2009, ApJ, 691, 1307

Hoshino, A., et al. 2010, PASJ, 62, 371

Ishisaki, Y., et al. 2007, PASJ, 59, 113

Kawaharada, M., et al. 2010, ApJ, 714, 423

Kalberla, P. M. W., Burton, W. B., Hartmann, D., et al. 2005, A\&A, 440, 775

Komatsu, E., et al. 2011, ApJS, 192, 18
Koyama, K., et al. 2007, PASJ, 59, 23

Kushino, A., Ishisaki, Y., Morita, U., Yamasaki, N. Y., Ishida, M., Ohashi, T., \& Ueda, Y. 2002, PASJ, 54, 327

Markevitch, M. 1998, ApJ, 504, 27

Markevitch, M., Forman, W. R., Sarazin, C. L., \& Vikhlinin, A. 1998, ApJ, 503, 77

Markevitch, M., et al. 2000, ApJ, 541, 542

Markevitch, M., \& Vikhlinin, A. 2007, Phys. Rep., 443, 1

Mitsuda, K., et al. 2010, Proc. SPIE, 7732,

Nagai, D., Kravtsov, A. V., \& Vikhlinin, A. 2007, ApJ, 668, 1

Nagai, D. and Lau, E. T. 2011, ApJL in press, arXiv:1103.0280

Okabe, N., \& Umetsu, K. 2008, PASJ, 60, 345

Pratt, G. W., Böhringer, H., Croston, J. H., Arnaud, M., Borgani, S., Finoguenov, A., \& Temple, R. F. 2007, A\&A, 461, 71

Pratt, G. W. and Arnaud, M. and Piffaretti, R. and Böhringer, H. and Ponman, T. J. and Croston, J. H. and Voit, G. M. and Borgani, S. and Bower, R. G. 2010, A\&A, 511A,85P

Piffaretti, R., \& Valdarnini, R. 2008, A\&A, 491, 71

Reiprich, T. H., et al. 2009, A\&A, 501, 899

Rudd,D.H., \& Nagai,D., 2009, ApJ, 701, L16

Serlemitsos, P. J., et al. 2007, PASJ, 59, 9

Simionescu, A., et al. 2011, Science, 331, 1576

Takei, Y., et al. 2008, ApJ, 680, 1049

Tamura, T., et al. 2008, PASJ, 60, 695

Tawa, N., et al. 2008, PASJ, 60, 11

Vikhlinin, A., Markevitch, M., Murray, S. S., Jones, C., Forman, W., \& Van Speybroeck, L. 2005, ApJ, 628, 655

Vikhlinin, A., Kravtsov, A., Forman, W., Jones, C., Markevitch, M., Murray, S. S., \& Van Speybroeck, L. 2006, ApJ, 640, 691

Voit, G. M., Balogh, M. L., Bower, R. G., Lacey, C. G., \& Bryan, G. L. 2003, ApJ, 593, 272

Voit,G,M.,2005,RvMP,77,207

Zhang, Y.-Y., Böhringer, H., Finoguenov, A., Ikebe, Y., Matsushita, K., Schuecker, P., Guzzo, L., \& Collins, C. A. 2006, A\&A, 456, 55

Umetsu, K., et al. 2009, ApJ, 694, 1643

Urban, O., Werner, N., Simionescu, A., Allen, S. W., Böhringer, H. 2011, MNRAS, 546 